\title{
DIFFRACTION OF COMPATIBLE RANDOM SUBSTITUTIONS IN ONE DIMENSION
}

\author{
MICHAEL BAAKE, TIMO SPINDELER, AND NICOLAE STRUNGARU
}

\begin{abstract}
As a guiding example, the diffraction measure of a random local mixture of the two classic Fibonacci substitutions is determined and reanalysed via self-similar measures of Hutchinson type, defined by a finite family of contractions. Our revised approach yields explicit formulas for the pure point and the absolutely continuous parts, as well as a proof for the absence of singular continuous components. This approach is then extended to the family of random noble means substitutions and, as an example with an underlying 2-adic structure, to a locally randomised version of the period doubling chain. As a first step towards a more general approach, we interpret our findings in terms of a disintegration over the Kronecker factor, which is the maximal equicontinuous factor of a covering model set.
\end{abstract}

\section{INTRODUCTION}

In general, the structure of systems with pure point diffraction is rather well understood $[11,38]$. Due to recent progress, see $[2,3,5]$ and references therein, also the situation for various systems with diffraction spectra of mixed type has improved. Still, the understanding of mixed spectra in the presence of entropy is only at its beginning and it is desirable to work out further concrete examples. Of particular interest, both theoretically and for applications to crystallography and physics, are cases that combine randomness with the presence of longrange aperiodic order, which means we will be looking for systems with non-trivial point spectrum in the presence of positive entropy.

In 1989, Godrèche and Luck [22] introduced a (locally) randomised extension of the two classic and well-studied Fibonacci substitutions, which individually, as well as under global mixtures, define the same hull. This is to say that any $S$-adic type sequence in these two substitutions will not result in a change of the hull it defines. In contrast, the local mixture, which emerges by deciding randomly, for each single letter, which of the two substitutions to apply (see Definition 4.1 for details), results in a much larger hull with positive entropy. Reference [22] contains first results on the topological entropy and the spectral type of the diffraction measure for the associated point sets. More precisely, the authors computed the set of Bragg peaks and argued that the diffraction measure is of mixed type, consisting of a pure point and an absolutely continuous part. Important steps towards a proof were given in $[32,33]$, though the absence of singular continuous components remained unproved.

The purpose of this paper is to present a closed expression for the formula of the diffraction intensities, and to give a proof for the fact that the diffraction measure is indeed of the expected mixed type, without singular continuous part. Later, we will consider various 
generalisations of this example, for instance by regarding the so-called noble means families, each consisting of finitely many primitive substitution rules that individually all define the same two-sided discrete dynamical hull, as does their global mixture. These cases had previously been considered in [33]. Here, we present a closed expression for the entire diffraction measures of their locally randomised version. Clearly, several results are already contained in $[32,42]$, the proofs of which will not be repeated here; see also [33] as well as the brief treatment in [4, Ch. 11].

As an interesting generalisation, we also consider a locally randomised version of the period doubling chain, which is built from a compatible pair of constant-length substitutions. While this is a simpler situation in the deterministic setting, this is not so here: In fact, its treatment requires a cut and project scheme with internal group $\mathbb{Z}_{2}$, the 2 -adic integers, and is thus treated separately. The type of spectral result we obtain is nevertheless the same: Almost surely, with respect to the ergodic patch frequency measure on the stochastic hull, the diffraction measure is of mixed type, with a pure point part that resembles the deterministic chain and an absolutely continuous part, but no singular continuous one.

The paper is organised as follows. We begin with a brief review of the deterministic Fibonacci tiling in Section 2, tailored to our later needs. Here, we also recall some now classic notions from the theory of aperiodic order, in particular its central tool, the cut and project method. To avoid unnecessary repetitions, we assume the interested reader to consult the recent monograph [4] for details. Section 3 is devoted to the construction and basic properties of the random Fibonacci inflation, which is followed by an extension to the family of random noble means inflations in Section 4. Up to this point, our entire treatment does not need any abstract tools from the theory of locally compact Abelian groups.

This changes when we turn our attention to an example from the class of constant-length

substitutions, which we keep separate in order not to overburden the exposition with a more abstract setting at the start. Here, we first recall the basic results for the deterministic period doubling chain in Section 5, then extending it to a randomised version in Section 6. Finally, looking back at the two types of examples, we identify one common structure in Section 7 , where we harvest an interesting connection between random substitutions and the theory of iterated functions systems, most notably Elton's ergodic theorem [19]. As a result, for all random substitutions discussed in this paper, the topological point spectrum is trivial, but one has a nice disintegration formula over the Kronecker factor, the latter emerging as the maximal equicontinuous factor of a covering model set. Also, the discontinuous eigenfunctions become continuous on a subset of the hull of full measure. A brief outlook concludes our exposition, and is followed by an appendix that proves some tricky, but often needed, approximation results for the autocorrelation.

\section{Deterministic Fibonacci tiling}

Before we investigate random substitutions, let us recall a paradigmatic deterministic case; see $[4,32]$ for a detailed exposition. Consider the binary alphabet $\mathcal{A}=\{a, b\}$ and the Fibonacci 
substitution given by $\zeta_{\mathrm{F}, 1}: a \mapsto a b, b \mapsto a$ or its variant $\zeta_{\mathrm{F}, 0}: a \mapsto b a, b \mapsto a$. Both share the same substitution matrix and also define the same symbolic hull $\mathbb{X}$, see $[4$, Ex. 4.6 and Rem. 4.6], wherefore we call them compatible.

Here, we are mainly interested in the geometric counterpart $\mathbb{Y}$ of $\mathbb{X}$ that emerges as the hull of the Fibonacci tilings, where $a$ and $b$ are tiles (intervals in our case) with natural lengths. As in [4], we use length $\tau=(1+\sqrt{5}) / 2$ for $a$ and 1 for $b$. This gives a topological dynamical system $(\mathbb{Y}, \mathbb{R})$ under the translation action of $\mathbb{R}$ that is strictly ergodic, with pure point spectrum. The pure-pointedness equivalently applies to both the diffraction and the dynamical spectrum $[29,6]$. In terms of diffraction, the spectral properties can be summarised as follows; see $[24,4]$ and references therein for proofs.

Theorem 2.1. Let $\mathbb{Y}$ be the geometric hull of the Fibonacci tiling system, with prototiles of length $\tau$ for type $a$ and 1 for type $b$. Then, the topological dynamical system $(\mathbb{Y}, \mathbb{R})$ is strictly ergodic and has pure point dynamical spectrum.

Now, fix some $\mathcal{T} \in \mathbb{Y}$ and let $\Lambda=\Lambda_{a} \dot{\cup} \Lambda_{b}$ be the corresponding set of left endpoints of the tiles in $\mathcal{T}$. Then, the weighted Dirac comb $\omega=u_{a} \delta_{\Lambda_{a}}+u_{b} \delta_{\Lambda_{b}}$, with any fixed pair of weights $u_{a}, u_{b} \in \mathbb{C}$, is pure point diffractive. Its autocorrelation is given by

$$
\gamma=\sum_{z \in \Lambda-\Lambda} \sum_{\alpha, \beta \in\{a, b\}} \overline{u_{\alpha}} \eta_{\alpha \beta}(z) u_{\beta} \delta_{z}
$$

where $\eta_{\alpha \beta}(z)=\operatorname{dens}\left(\Lambda_{\alpha} \cap\left(\Lambda_{\beta}-z\right)\right)$, and the diffraction measure reads

$$
\widehat{\gamma}=\sum_{k \in \mathbb{Z}[\tau] / \sqrt{5}} I(k) \delta_{k}
$$

where $I(k)=\left|u_{a} A_{\Lambda_{a}}(k)+u_{b} A_{\Lambda_{b}}(k)\right|^{2}$, with the Fourier-Bohr coefficients of $t+\Lambda_{\alpha}$, for fixed $t \in \mathbb{R}$ and $\alpha \in\{a, b\}$, being given by

$$
A_{t+\Lambda_{\alpha}}(k)=\lim _{r \rightarrow \infty} \frac{1}{2 r} \sum_{x \in\left(t+\Lambda_{\alpha}\right) \cap[-r, r]} \mathrm{e}^{-2 \pi \mathrm{i} k x} .
$$

In particular, $\gamma$ and $\Lambda-\Lambda$, as well as $\hat{\gamma}$ and $I(k)$, are independent of $\mathcal{T}$, while the FourierBohr coefficients do depend on the chosen element, but converge uniformly in $t$.

Remark 2.2. The Fourier-Bohr coefficients in Theorem 2.1 exist for all $k \in \mathbb{R}$, but vanish unless $k \in \mathbb{Z}[\tau] / \sqrt{5}$. For any $k$ of the latter type, the map $\Lambda \mapsto A_{\Lambda}(k)=A_{\Lambda_{a}}(k)+A_{\Lambda_{b}}(k)$ defines a continuous eigenfunction $[24,30]$ for $(\mathbb{Y}, \mathbb{R})$, with

$$
A_{t+\Lambda}(k)=\mathrm{e}^{-2 \pi \mathrm{i} k t} A_{\Lambda}(k)
$$

provided $A_{\Lambda}(k) \neq 0$. This connection can be used to show that the dynamical spectrum is given by $\mathbb{Z}[\tau] / \sqrt{5}$; see $[4$, Sec. 9.4.1] for details on extinctions. The latter precisely occur for $k=\ell \tau$ with $\ell \in \mathbb{Z} \backslash\{0\}$ provided that $u_{a}=u_{b}$. For a generic choice of the weights, no extinctions are present. 
Via the projection method, compare [4, Sec. 7], the elements of $\mathbb{Y}$ can be described as (translations of) regular model sets within the cut and project scheme, or CPS for short, $(\mathbb{R}, \mathbb{R}, \mathcal{L})$. This is briefly summarised by the diagram

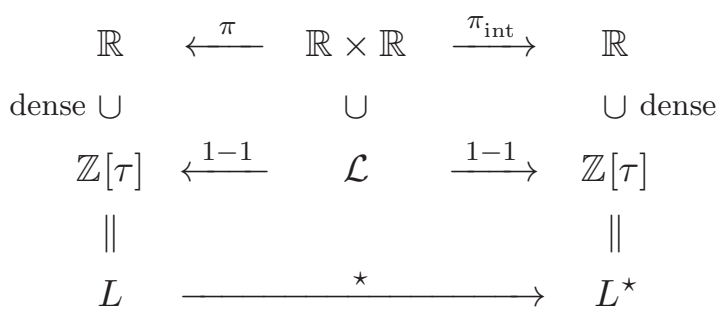

where the lattice, $\mathcal{L}$, is given by

$$
\mathcal{L}:=\left\{\left(x, x^{\star}\right) \mid x \in \mathbb{Z}[\tau]\right\}
$$

and the $\star$-map is algebraic conjugation in $\mathbb{Q}(\sqrt{5})$, as defined by the unique extension of $\sqrt{5} \mapsto-\sqrt{5}$ to a field automorphism of $\mathbb{Q}(\sqrt{5})$. Concretely, consider the fixed point of $\zeta_{\mathrm{F}, 1}^{2}$ with legal seed $b \mid a$. The corresponding tiling $\mathcal{T}$ leads to $\Lambda=\Lambda_{a} \dot{\cup} \Lambda_{b}$ together with

$$
\Lambda_{a}=\curlywedge([\tau-2, \tau-1)), \quad \Lambda_{b}=\curlywedge([-1, \tau-2)) \text { and } \Lambda=\curlywedge([-1, \tau-1)),
$$

where $\curlywedge(W):=\left\{x \in \mathbb{Z}[\tau] \mid x^{\star} \in W\right\}$; see [4, Ex. 7.3] for details, in particular for the changes in the windows if we would work with the other possible seed, $a \mid a$. In the formulation of Theorem 2.1, the Fourier-Bohr coefficients of this particular $\Lambda$, for $k \in \mathbb{Z}[\tau] / \sqrt{5}$, read

$$
A_{\Lambda_{a}}(k)=\frac{1}{\sqrt{5}} \int_{\tau-2}^{\tau-1} \mathrm{e}^{2 \pi \mathrm{i} k^{\star} y} \mathrm{~d} y \quad \text { and } \quad A_{\Lambda_{b}}(k)=\frac{1}{\sqrt{5}} \int_{-1}^{\tau-2} \mathrm{e}^{2 \pi \mathrm{i} k^{\star} y} \mathrm{~d} y .
$$

When working with the other inflation rule, based on $\zeta_{\mathrm{F}, 0}^{2}$, completely analogous formulas can be derived; see Remark 3.8 below for further comments and [4] for the general theory.

For the rule we selected here, similar expressions can also be derived for other elements of $\mathbb{Y}$; see $[4,32]$ for more. In this setting, one can express the pair correlation coefficients $\eta_{\alpha \beta}(z)$ from Theorem 2.1 as

$$
\eta_{\alpha \beta}(z)=\operatorname{dens}(\Lambda) \frac{\operatorname{vol}\left(W_{\alpha} \cap\left(W_{\beta}-z^{\star}\right)\right)}{\operatorname{vol}(W)}=\frac{1}{\sqrt{5}} \int_{\mathbb{R}} 1_{W_{\alpha}}(y) 1_{W_{\beta}-z^{\star}}(y) \mathrm{d} y,
$$

where the $W_{\alpha}$ with $\alpha \in\{a, b\}$ are the windows for the model set description from Eq. (2).

Due to the Pisot nature of the golden ratio, $\tau$, one can go one step further and consider a modified (or deformed) hull $\tilde{\mathbb{Y}}$ that emerges from $\mathbb{X}$ by taking $a$ and $b$ type intervals of lengths

$$
\ell_{a}=\tau+\rho(1-\tau) \text { and } \ell_{b}=1+\rho,
$$

respectively, where $\rho \in(-1, \tau+1)$ is a real parameter. This choice is made so that the average tile length, and hence also the density of left endpoints, is the same for $\mathbb{Y}$ and $\tilde{\mathbb{Y}}$. Since the elements of $\tilde{\mathbb{Y}}$ are always considered as tilings with two distinct prototiles, even if they have the same length (as happens for $\rho=\tau^{-2}$ ), the dynamical systems $(\mathbb{Y}, \mathbb{R})$ and $(\tilde{\mathbb{Y}}, \mathbb{R})$ are topologically conjugate in this setting. This follows from the description of the elements 
of $\tilde{\mathbb{Y}}$ as deformed model sets, see [14, 7], and is in line with the general analysis of [17]. In terms of the diffraction, and in complete analogy to [4, Ex. 9.9], the result is the following.

Corollary 2.3. Consider the dynamical system $(\tilde{\mathbb{Y}}, \mathbb{R})$ with parameter $\rho \in(-1, \tau+1)$ as above. Select any tiling $\mathcal{T}^{\prime} \in \tilde{\mathbb{Y}}$ and consider the corresponding point set $\Lambda^{\prime}=\Lambda_{a}^{\prime} \dot{\cup} \Lambda_{b}^{\prime}$ of left endpoints. Then, the Dirac comb $\omega^{\prime}=u_{a} \delta_{\Lambda_{a}^{\prime}}+u_{b} \delta_{\Lambda_{b}^{\prime}}$ is pure point diffractive, with diffraction measure

$$
\widehat{\gamma^{\prime}}=\sum_{k \in \mathbb{Z}[\tau] / \sqrt{5}} I^{\prime}(k) \delta_{k}
$$

where $I^{\prime}(k)=\left|u_{a} A_{\Lambda_{a}}^{\prime}(k)+u_{b} A_{\Lambda_{b}}^{\prime}(k)\right|^{2}$, and the Fourier-Bohr coefficients are defined in complete analogy to Theorem 2.1. As before, the diffraction measure is independent of $\mathcal{T}^{\prime}$, while the Fourier-Bohr coefficients do depend on it, but converge uniformly.

If we select $\mathcal{T}^{\prime}$ as the deformed version of the fixed point tiling from Eq. (2), we obtain an analogue of Eq. (3) for the Fourier-Bohr coefficients. With $\operatorname{sinc}(x):=\frac{\sin (x)}{x}$, one finds

$$
A_{\Lambda_{a}}^{\prime}(k)=\frac{1}{\sqrt{5}} \int_{\tau-2}^{\tau-1} \mathrm{e}^{2 \pi \mathrm{i}\left(k^{\star}-\rho k\right) y} \mathrm{~d} y=\frac{\mathrm{e}^{\pi \mathrm{i}(2 \tau-3)\left(k^{\star}-\rho k\right)}}{\sqrt{5}} \operatorname{sinc}\left(\pi\left(k^{\star}-\rho k\right)\right)
$$

and

$$
A_{\Lambda_{b}}^{\prime}(k)=\frac{1}{\sqrt{5}} \int_{-1}^{\tau-2} \mathrm{e}^{2 \pi \mathrm{i}\left(k^{\star}-\rho k\right) y} \mathrm{~d} y=\frac{\mathrm{e}^{\pi \mathrm{i}(\tau-3)\left(k^{\star}-\rho k\right)}}{\tau \sqrt{5}} \operatorname{sinc}\left(\pi(\tau-1)\left(k^{\star}-\rho k\right)\right),
$$

which sum to

$$
A_{\Lambda}^{\prime}(k)=A_{\Lambda_{a}}^{\prime}(k)+A_{\Lambda_{b}}^{\prime}(k)=\frac{\tau \mathrm{e}^{-\pi \mathrm{i} \tau^{-2}\left(k^{\star}-\rho k\right)}}{\sqrt{5}} \operatorname{sinc}\left(\pi \tau\left(k^{\star}-\rho k\right)\right) .
$$

For $\rho=0$, this gives back the previous expressions.

Remark 2.4. Our derivation employed the method of deformed model sets, see [14] for details, but the special cases at hand can alternatively be written as model sets with a new lattice that emerges from the original one by a shear in the physical direction.

Moreover, due to our choice of tile lengths according to Eq. (4), the dynamical spectrum remains pure point and is always given by $\mathbb{Z}[\tau] / \sqrt{5}$, as in Remark 2.2. In particular, all deformed model sets in our class here define dynamical systems that are metrically isomorphic by the Halmos-von Neumann theorem, and are even topologically conjugate to each other as mentioned earlier.

\section{RANDOM FibONACCI TILING}

The topological dynamical system $(\mathbb{X}, S)$ from the previous section has zero entropy, because the word complexity is linear. Alternatively, this fact also follows from [9]. Let us now generalise the Fibonacci substitution and construct random Fibonacci sets with positive entropy that still show long-range order $[22,31,36,33]$.

To this end, let $p \in[0,1]$ be a fixed probability and set $q=1-p$. If $\mathcal{A}=\{a, b\}$ is our binary alphabet as before, we use $\mathcal{A}^{*}$ to denote the set of finite words with letters from $\mathcal{A}$. This is 
a monoid under the concatenation of words as multiplication. Now, the random Fibonacci substitution is the endomorphism $\zeta_{\mathrm{F}}: \mathcal{A}^{*} \rightarrow \mathcal{A}^{*}$ defined by

$$
\zeta_{\mathrm{F}}:\left\{\begin{array}{l}
a \mapsto \begin{cases}b a, & \text { with probability } p, \\
a b, & \text { with probability } q,\end{cases} \\
b \mapsto a .
\end{array}\right.
$$

Here, given a word $w \in \mathcal{A}^{*}$, we independently apply $\zeta_{\mathrm{F}}$ to each letter of $w$, which reflects the endomorphism property. Consequently, $\zeta_{\mathrm{F}}(a)$ as well as $\zeta_{\mathrm{F}}(b)$, and hence $\zeta_{\mathrm{F}}(w)$, have to be considered as random variables, each with finitely many possible realisations in our case. A more general definition will be given in the next section, but is not needed here.

Of course, the term 'random' is only justified for $p \in(0,1)$, while $p=0$ and $p=1$ correspond to the two deterministic limiting cases from the previous section. The latter are primitive, with the same substitution matrix, which is inherited by the random version. To proceed, we need to adjust some definitions from symbolic dynamics to the stochastic situation, where we follow the approach of $[32,33]$ which extends earlier work $[22,31]$; see also [40, 23].

Definition 3.1. Let $\zeta_{\mathrm{F}}$ be the primitive random substitution from Eq. (5). Then, for any $v, w \in \mathcal{A}^{*}$ and $k \in \mathbb{N}$, we use $v \varangle \zeta_{\mathrm{F}}^{k}(w)$ to express that $v$ is a subword of at least one realisation of $\zeta_{\mathrm{F}}^{k}(w)$. A word $w \in \mathcal{A}^{*}$ is called $\zeta_{\mathrm{F}}$-legal if there is a $k \in \mathbb{N}$ such that $w \triangleleft \zeta_{\mathrm{F}}^{k}(a)$. The $\zeta_{\mathrm{F}}$-dictionary is defined as

$$
\mathcal{D}_{\zeta_{\mathrm{F}}}:=\left\{w \in \mathcal{A}^{*} \mid w \text { is } \zeta_{\mathrm{F}^{-}} \text {legal }\right\},
$$

while the two-sided discrete stochastic hull of $\zeta_{\mathrm{F}}$ is denoted as

$$
\mathbb{X}_{\zeta_{\mathrm{F}}}:=\left\{w \in \mathcal{A}^{\mathbb{Z}} \mid \mathfrak{F}(\{w\}) \subseteq \mathcal{D}_{\zeta_{\mathrm{F}}}\right\}
$$

where $\mathfrak{F}(\{w\})$ is the set of all finite subwords of $w$.

Obviously, one can replace $\zeta_{\mathrm{F}}$ by any other primitive random substitution $\varrho$; see Definition 4.1 below. Let us recall a result on the entropy of the discrete stochastic hull, which illustrates a fundamental difference from the deterministic case.

Fact $3.2([36,32])$. For $\zeta_{\mathrm{F}}$ from Eq. (5) and any $p \in(0,1)$, the topological entropy of the dynamical system $\left(\mathbb{X}_{\zeta_{\mathrm{F}}}, \mathbb{Z}\right)$ is given by $s=\sum_{\ell=2}^{\infty} \frac{\log (\ell)}{\tau^{\ell+2}} \approx 0.444398725$.

3.1. Tiling picture. To continue, we need to consider the geometric counterpart of $\mathbb{X}_{\zeta_{\mathrm{F}}}$. In a first step, we again replace letters by intervals of length $\tau$ (for $a$ ) and 1 (for $b$ ), and take the left endpoints of all intervals to obtain Delone sets. From now on, we will identify the tiling picture with the Delone picture that emerges from taking the left endpoints, possibly marked by the tile type, as this gives a bijection between the two sets of objects. This procedure turns each element $w \in \mathbb{X}_{\zeta_{\mathrm{F}}}$ into a point set $\Lambda_{w}$, where we agree to map the marker (origin) of $w$ to 0 . This means that each point set $\Lambda_{w}$ is a Delone set that contains 0 , and the collection of these sets constitutes the punctured continuous hull $\mathbb{Y}_{0, \zeta_{\mathrm{F}}}$. 


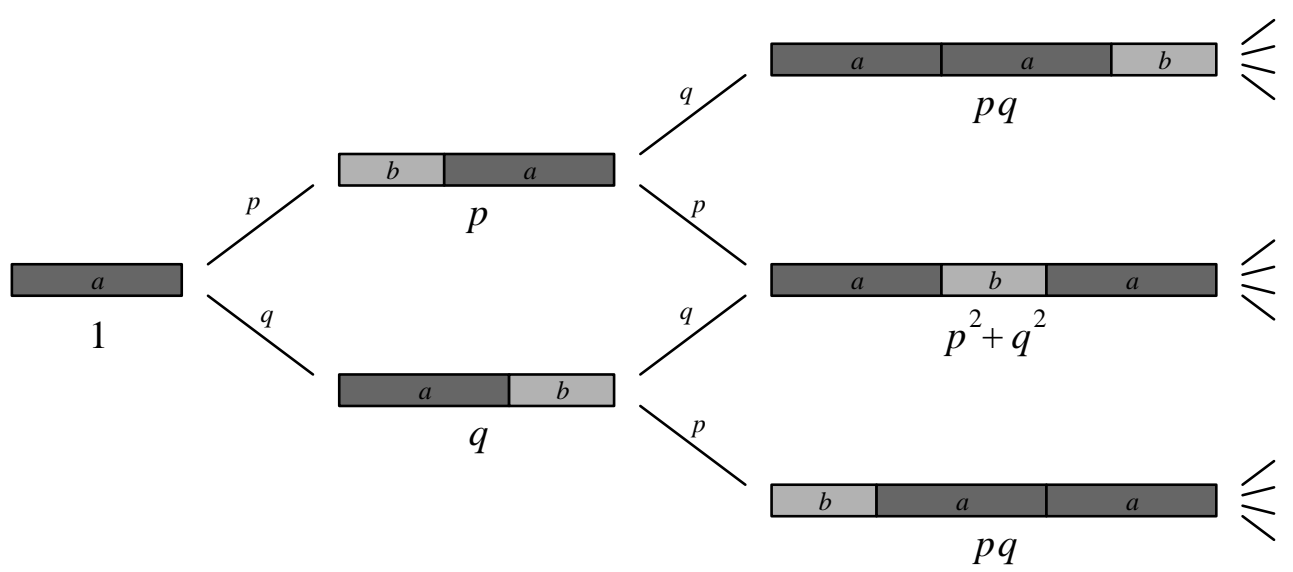

Figure 1. Illustration of the first steps of the random Fibonacci inflation rule with natural interval lengths. It shows the transition probabilities as well as the probabilities for the first exact inflation patches. The probability measure induced by this process on the (one-sided) infinite inflation patches is compatible with the patch frequency measure obtained from Perron-Frobenius theory; compare [32, 23].

At this stage, there is no translation action of $\mathbb{R}$ on this hull. To construct a proper dynamical system, we need the full continuous hull $\mathbb{Y}_{\zeta_{F}}$, which is obtained as the smallest collection of Delone sets that contains $\mathbb{Y}_{0, \zeta_{\mathrm{F}}}$ and is closed under the (continuous) translation action of $\mathbb{R}$. Then, $\left(\mathbb{Y}_{\zeta_{F}}, \mathbb{R}\right)$ is a topological dynamical system that is topologically conjugate to the suspension of the discrete system with a roof function that reflects the tile lengths. Clearly, one has $\mathbb{Y}_{0, \zeta_{\mathrm{F}}}=\left\{y \in \mathbb{Y}_{\zeta_{\mathrm{F}}} \mid 0 \in y\right\}$.

The elements of $\mathbb{Y}_{\zeta_{\mathrm{F}}}$ can be described as subsets of (translates of) model sets. It suffices to see this for the elements of $\mathbb{Y}_{0, \zeta_{F}}$. The idea is to use the positions of the windows from the deterministic case relative to each other to derive a covering window $W$ with $\Lambda \subset \curlywedge(W)$; see Section 7 for a detailed derivation of this property.

Remark 3.3. Recall that, in the deterministic setting, the discrete hull of a primitive substitution can be constructed via the fixed point of the substitution. In the stochastic situation, there is no direct analogue of a fixed point. However, it is possible to modify this approach. To do so, define

$$
X_{\zeta_{\mathrm{F}}}:=\left\{w \in \mathcal{A}^{\mathbb{Z}} \mid w \text { is an accumulation point of }\left(\zeta_{\mathrm{F}}^{k}(a \mid a)\right)_{k \in \mathbb{N}_{0}}\right\},
$$

where 'accumulation point' is meant in the sense of one for any of the possible realisations of the random substitution sequence. Then, the hull $\mathbb{X}_{\zeta_{\mathrm{F}}}$ from above is the smallest closed and shift-invariant subset of $\mathcal{A}^{\mathbb{Z}}$ with $X_{\zeta_{\mathrm{F}}} \subseteq \mathbb{X}_{\zeta_{\mathrm{F}}}$; see [32, Prop. 2.22]. The geometric realisations of elements of $X_{\zeta_{\mathrm{F}}}$ are called generating random Fibonacci sets. 
Proposition 3.4 ([32, Prop. 5.21]). Let $\Lambda$ be any of the generating random Fibonacci sets from Remark 3.3. Then, one has $\Lambda \subset \curlywedge(W)$ with covering window $W=[-\tau, \tau]$, where $\operatorname{dens}(\curlywedge(W))=\frac{2 \tau}{\sqrt{5}}=2 \operatorname{dens}(\Lambda)$.

Now, we want to determine the diffraction measure for the system defined by $\zeta_{\mathrm{F}}$, which means to determine the average over the diffraction measures of the elements of $\mathbb{Y}_{\zeta_{\mathrm{F}}}$ with respect to an invariant measure on it. The latter is chosen as the patch frequency measure, denoted by $\nu_{\mathrm{pf}}$, with frequencies defined via a van Hove sequence $\left(B_{n}\right)_{n \in \mathbb{N}}$ of growing intervals that are centred at the origin, so $B_{n} \subset B_{n+1}$ for all $n$ and $\operatorname{vol}\left(B_{n}\right) \stackrel{n \rightarrow \infty}{\longrightarrow} \infty$. Note that $\nu_{\text {pf }}$ is a completely natural choice, and is both translation invariant and ergodic [33]. In fact, it has the nice feature that the unique measure induced by it on the subset $X_{\zeta_{\mathrm{F}}}$ via filtration is precisely the measure defined directly on this subset by the random inflation procedure according to Figure 1; see [23] for details.

Consider an individual $\Lambda \in \mathbb{Y}_{\zeta_{\mathrm{F}}}$. Its diffraction measure (with respect to the same van Hove sequence), which really is the diffraction measure of the Dirac comb $\delta_{\Lambda}$, is given by

$$
\widehat{\gamma_{\Lambda}}=\lim _{n \rightarrow \infty} \frac{1}{\operatorname{vol}\left(B_{n}\right)}\left|\sum_{x \in \Lambda_{n}} \mathrm{e}^{-2 \pi \mathrm{i} k x}\right|^{2}=\lim _{n \rightarrow \infty} \frac{1}{\operatorname{vol}\left(B_{n}\right)}\left|X_{n}(k)\right|^{2},
$$

with $\Lambda_{n}=\Lambda \cap B_{n}$ and the exponential sums $X_{n}(k):=\sum_{x \in \Lambda_{n}} \mathrm{e}^{-2 \pi \mathrm{i} k x}$. The limit is taken in the vague topology. It exists, and is the same, for $\nu_{\mathrm{pf}}$-almost all $\Lambda \in \mathbb{Y}_{\zeta_{\mathrm{F}}}$, both as a consequence of the ergodic theorem. Let us use $\widehat{\gamma}$ to denote this limit, which is the diffraction measure of the measure-theoretic dynamical system $\left(\mathbb{Y}_{\zeta_{\mathrm{F}}}, \mathbb{R}, \nu_{\mathrm{pf}}\right)$. As such, it can be determined as an $\nu_{\mathrm{pf}}$-average over the individual diffraction measures $\widehat{\gamma_{\Lambda}}$.

Now, to calculate $\widehat{\gamma}$, we actually do not need to take an average over (almost) all individual diffraction measures $\widehat{\gamma_{\Lambda}}$, but only over a suitable subset. A good choice of the latter will then actually allow us to find the corresponding Eberlein decomposition as well. A justification of this step follows later, in Section 7. Here, we work with one-sided tilings (starting from the origin) and approach them by the family of exact inflation patches, viewed as random variables. Denoting the corresponding random exponential sums for $n$ inflation steps by $\mathcal{X}_{n}(k)$, we can determine their probabilistic weights by the corresponding random concatenation rule; see [22, Eq. 5.9]. This gives the recursion

$$
\mathcal{X}_{n}(k)= \begin{cases}\mathcal{X}_{n-2}(k)+\mathrm{e}^{-2 \pi \mathrm{i} k \tau^{n-2}} \mathcal{X}_{n-1}(k), & \text { with probability } p, \\ \mathcal{X}_{n-1}(k)+\mathrm{e}^{-2 \pi \mathrm{i} k \tau^{n-1}} \mathcal{X}_{n-2}(k), & \text { with probability } q,\end{cases}
$$

together with $\mathcal{X}_{0}(k)=1$ and $\mathcal{X}_{1}(k)=1$. Note that $\left(\mathcal{X}_{n}(k)\right)_{n \in \mathbb{N}}$ can be seen as the subsequence of $\left(X_{m}(k)\right)_{m \in \mathbb{N}}$ that corresponds to exact random Fibonacci inflation patches.

Remark 3.5. Here and below, we work with the left endpoints of each tile. Consequently, the initial conditions $\mathcal{X}_{0}$ and $\mathcal{X}_{1}$ differ from the ones used in [22], where Godrèche and Luck chose the right endpoints of each tile instead. This does not affect the final result. 
Let us now use $\mathbb{E}$ for the average over the exact inflation patches, weighted with their appropriate probabilities. Then, we obtain

$$
\mathbb{E}\left(\widehat{\gamma_{\Lambda}}\right)=\lim _{n \rightarrow \infty} \frac{\left|\mathbb{E}\left(\mathcal{X}_{n}\right)\right|^{2}}{L_{n}}+\lim _{n \rightarrow \infty} \frac{\operatorname{Var}\left(\mathcal{X}_{n}\right)}{L_{n}}=: \widehat{\gamma_{1}}+\widehat{\gamma_{2}}
$$

where $L_{n}=\tau^{n}$ is the length of the level- $n$ random inflation tiling patch. It is the same for all realisations. The existence of the two limits was constructively shown in $[22,32,33]$. It was also shown there that $\widehat{\gamma_{2}}$ is absolutely continuous. One aim of this paper is to complete the spectral analysis of this case by showing that $\widehat{\gamma_{1}}$ is pure point, which implies the absence of singular continuous components.

To this end, let $\mathcal{M}_{n}$ denote the (finite) random Dirac comb that underlies $\mathcal{X}_{n}$, so $\mathcal{X}_{n}=\widehat{\mathcal{M}_{n}}$. With initial conditions $\mathcal{M}_{0}=\mathcal{M}_{1}=\delta_{0}$, the counterpart to Eq. (7) reads

$$
\mathcal{M}_{n}= \begin{cases}\mathcal{M}_{n-2}+\delta_{\tau^{n-2}} * \mathcal{M}_{n-1}, & \text { with probability } p, \\ \mathcal{M}_{n-1}+\delta_{\tau^{n-1}} * \mathcal{M}_{n-2}, & \text { with probability } q,\end{cases}
$$

and we know the following property from $[32,33]$ and Proposition 3.4.

Fact 3.6. For any $n \in \mathbb{N}$, all realisations of the random Dirac comb $\mathcal{M}_{n}$ have support in the finite point set $\curlywedge(W) \cap\left[0, L_{n}\right)$, with $L_{n}=\tau^{n}$ as before.

3.2. Averages and weight functions. Define the uniformly discrete point set

$$
\Lambda_{\geqslant 0}:=\curlywedge(W) \cap \mathbb{R}_{\geqslant 0}=\{x \in \mathcal{\curlywedge}(W): x \geqslant 0\}
$$

and observe that we may identify $\mathcal{M}_{n}$ with a random variable with values in $\Omega:=\{0,1\}^{\Lambda \geqslant 0}$. Note that $\Omega$ is a compact space, equipped with the standard product topology. In this picture, the realisations of $\mathcal{M}_{n}$ are sequences of the form $\left(m_{x}\right)_{x \in \Lambda_{\geqslant 0}}$ with $m_{x} \in\{0,1\}$ and $m_{x}=0$ for all $x \geqslant L_{n}$, the latter due to our setting with left endpoints of the tiles as markers. Let $\vartheta_{n}$ denote the corresponding (discrete) probability distribution on $\Omega$.

Lemma 3.7. Let $p$ and $q$ be the probabilities from Eq. (5), and assume that $0<p<1$. Then, the sequence $\left(\vartheta_{n}\right)_{n \in \mathbb{N}}$ of probability measures on $\Omega$ is weakly converging.

Proof. The claimed convergence, by standard arguments, is equivalent to the convergence of $\left(\vartheta_{n}(Z)\right)_{n \in \mathbb{N}}$ for every cylinder set $Z$ that is specified at a finite set of positions in $\Lambda_{\geqslant 0}$. A simple inclusion-exclusion argument shows that this is equivalent to the convergence of $\vartheta_{n}\left(\left\{m_{x}=1\right.\right.$ for $\left.\left.x \in F\right\}\right)$ for any finite $F \subset \Lambda_{\geqslant 0}$. Let

$$
g^{(n)}(x)=\vartheta_{n}\left(\left\{m_{x}=1\right\}\right)=g_{a}^{(n)}(x)+g_{b}^{(n)}(x)
$$

be the occupation probability of position $x$ under $\vartheta_{n}$, split into those for type $a$ and $b$. For any $p \in[0,1]$, the random inflation (5) now implies the recursion

$$
\begin{aligned}
& g_{a}^{(n+1)}(x)=q g_{a}^{(n)}\left(\frac{x}{\tau}\right)+g_{b}^{(n)}\left(\frac{x}{\tau}\right)+p g_{a}^{(n)}\left(\frac{x-1}{\tau}\right), \\
& g_{b}^{(n+1)}(x)=p g_{a}^{(n)}\left(\frac{x}{\tau}\right)+q g_{a}^{(n)}\left(\frac{x-\tau}{\tau}\right),
\end{aligned}
$$


subject to the initial condition $g_{\alpha}^{(0)}(x)=\delta_{\alpha, a} \delta_{x, 0}$ and the general rule that, for any $\alpha \in\{a, b\}$ and all $n$, we have $g_{\alpha}^{(n)}(x)=0$ whenever $x \notin \Lambda_{\geqslant 0}$. At $x=0$, this implies

$$
\left(\begin{array}{l}
g_{a}^{(n+1)}(0) \\
g_{b}^{(n+1)}(0)
\end{array}\right)=\left(\begin{array}{ll}
q & 1 \\
p & 0
\end{array}\right)\left(\begin{array}{l}
g_{a}^{(n)}(0) \\
g_{b}^{(n)}(0)
\end{array}\right)
$$

where the matrix on the right-hand side is the (transpose of) a Markov matrix. It is primitive when $0<p<1$, which implies the convergence of the sequences $\left(g_{\alpha}^{(n)}(0)\right)_{n \in \mathbb{N}}$, with limits $g_{a}(0)=1 /(1+p)$ and $g_{b}(0)=p /(1+p)$.

Now, writing $\Lambda_{\geqslant 0}=\left\{0=x_{0}<x_{1}<x_{2}<\ldots\right\}$, the convergence of $\left(g_{\alpha}^{(n)}\left(x_{i}\right)\right)_{n \in \mathbb{N}}$ can be shown inductively in $i$, because the structure of Eq. (11) is such that, when $x>0$, the right-hand side only has arguments $y$ with $0 \leqslant y<x$, where the functions are either known to vanish or converge by induction. This shows the convergence of the marginals for all cylinder sets that are specified at a single location.

A similar argument, based on the propagation of prefix probabilities, also works for the cylinder sets specified on a finite set of positions. Here, given any finite set $\varnothing \neq F \subset \Lambda_{\geqslant 0}$, one chooses an integer $n$ such that the geometric realisation (as a patch) of any legal word of length $n$ is longer than the largest element of $F$. We consider this as a collection of prefix patches. Taking all possible inflations of these patches and sorting them according to the same prefix collection, we derive a transition matrix for the prefix collection under one inflation step, which is (the transpose of) a Markov matrix by construction. When $0<p<1$, this matrix is irreducible by standard arguments and cyclically primitive because the corresponding graph must contain a loop, which implies primitivity of the matrix.

Now, we equip the prefix patches with initial probabilities, for instance via a set of exact inflation patches of sufficient size (we know that this is possible because we only look at legal words of length $n$ ). No matter what these initial probabilities are, a repeated iteration of the primitive Markov matrix gives a converging sequence of prefix probability vectors, with the limit being independent of the initial choice. This finally implies the convergence of $\vartheta_{n}\left(\left\{m_{x}=1\right.\right.$ for $\left.\left.x \in F\right\}\right)$ as $n \rightarrow \infty$, and our claim follows.

Remark 3.8. It is easy to check that, with our initial condition, the iteration of Eq. (12) also converges for $p=0$, but not for $p=1$, where the iteration alternates between the vectors $(1,0)^{t}$ and $(0,1)^{t}$. These are the deterministic cases, where the inflation has a one-sided fixed point for $p=0$, but not for $p=1$. In the latter case, one has two fixed points for the square of the inflation instead. Here, one could 'restore' convergence by starting from the modified initial condition $g_{a}^{(0)}(0)=g_{b}^{(0)}(0)=\frac{1}{2}$.

Looking at the actual sequence of inflation words for $p=1$, however, reveals that only the first two positions alternate between $a b$ and $b a$, while all following positions are stable. This simply reflects the fact that we are working with a singular element of the discrete hull here, which is not relevant in the random situation. 
Let us continue with the random case, $0<p<1$, and set $g_{\alpha}(x)=\lim _{n \rightarrow \infty} g_{\alpha}^{(n)}(x)$. Then, Eq. (11) implies the exact renormalisation identities

$$
\begin{aligned}
& g_{a}(x)=q g_{a}\left(\frac{x}{\tau}\right)+g_{b}\left(\frac{x}{\tau}\right)+p g_{a}\left(\frac{x-1}{\tau}\right), \\
& g_{b}(x)=p g_{a}\left(\frac{x}{\tau}\right)+q g_{a}\left(\frac{x-\tau}{\tau}\right),
\end{aligned}
$$

together with $g_{\alpha}(x)=0$ for $\alpha \in\{a, b\}$ and all $x \notin \Lambda_{\geqslant 0}$. In fact, one has more.

Proposition 3.9. The renormalisation relations from Eq. (13), subject to the condition that $g_{\alpha}(x)=0$ for $\alpha \in\{a, b\}$ and all $x \notin \Lambda_{\geqslant 0}$, have a one-dimensional solution space, for any $p \in[0,1]$. In particular, there is precisely one solution with $g_{a}(0)=1 /(1+p)$.

Moreover, for each $n \in \mathbb{N}_{0}$, the support of the function $g^{(n)}$ from Eq. (10) is contained in the finite set $\Lambda^{(n)}:=\Lambda \cap\left[0, L_{n}\right)$, and the pointwise limits $g(x)=\lim _{n \rightarrow \infty} g^{(n)}(x)$ are uniform in the sense that $\max _{x \in \Lambda^{(n)}}\left|g(x)-g^{(n)}(x)\right| \stackrel{n \rightarrow \infty}{\longrightarrow} 0$.

Proof. The first claim really is a consequence of Lemma 3.7 and its proof. Indeed, taking the limit in Eq. (12) gives an eigenvector equation with eigenvalue 1. It is easy to check that the corresponding eigenspace is always one-dimensional, including the cases $p=0$ and $p=1$. Since all other values $g_{\alpha}(x)$ are determined recursively, our claim is obvious. The unique solution specified by the special value is the one we need for our further analysis.

The supporting set of $g^{(n)}=g_{a}^{(n)}+g_{b}^{(n)}$ follows from the recursion relations in Eq. (12) by induction. The claim on the convergence is obvious for the two deterministic cases, $p=0$ and $p=1$, because one then simply has $g^{(n)}(x)=g(x)$ on $\Lambda^{(n)}$. In general, the concatenation structure implies the recursion

$$
g^{(n+1)}(x)= \begin{cases}q g^{(n)}(x)+p g^{(n-1)}(x), & x \in \Lambda^{(n-1)}, \\ q g^{(n)}(x)+p g^{(n-1)}\left(x-\tau^{n-1}\right), & x \in \Lambda^{(n)} \backslash \Lambda^{(n-1)}, \\ p g^{(n)}\left(x-\tau^{n-1}\right)+q g^{(n-1)}\left(x-\tau^{n}\right), & x \in \Lambda^{(n+1)} \backslash \Lambda^{(n)} .\end{cases}
$$

This can now be used inductively to show that

$$
\lim _{n \rightarrow \infty} \max _{x \in \Lambda^{(n)}}\left|g(x)-g^{(n)}(x)\right|=0,
$$

the technical details of which are given in [42, Lemma 3.29]. This implies our claim.

3.3. Lift into internal space. Let $\vartheta$ be the probability measure on $\Omega$ that is the weak limit of the sequence $\left(\vartheta_{n}\right)_{n \in \mathbb{N}}$. This means that there is a random variable $\mathcal{M}$ with law $\vartheta$ such that $\left(\mathcal{M}_{n}\right)_{n \in \mathbb{N}}$ converges in distribution to $\mathcal{M}$, which in particular implies that

$$
\mathbb{E}(\mathcal{M})=\lim _{n \rightarrow \infty} \mathbb{E}\left(\mathcal{M}_{n}\right)=\sum_{x \in \Lambda_{\geqslant 0}} \vartheta\left(\left\{m_{x}=1\right\}\right) \delta_{x}
$$

with $\vartheta\left(\left\{m_{x}=1\right\}\right)=g_{a}(x)+g_{b}(x)$ as detailed above. Now, we want to determine the coefficients of $\mathbb{E}(\mathcal{M})$ more explicitly, for which we employ a representation within our CPS. 
To do so, we write $\mathbb{E}\left(\mathcal{M}_{n}\right)$ and $\mathbb{E}(\mathcal{M})$ as

$$
\mathbb{E}\left(\mathcal{M}_{n}\right)=\sum_{x \in \Lambda_{\geqslant 0}} h^{(n)}\left(x^{\star}\right) \delta_{x} \quad \text { and } \quad \mathbb{E}(\mathcal{M})=\sum_{x \in \Lambda_{\geqslant 0}} h\left(x^{\star}\right) \delta_{x}
$$

As suggested by our previous analysis, we write

$$
h^{(n)}=h_{a}^{(n)}+h_{b}^{(n)}
$$

with obvious meaning. Define now $\mu_{\alpha}^{(n)}=\frac{c}{F_{n}} \sum_{x \in \mathcal{\lambda}(W)} h_{\alpha}^{(n)}\left(x^{\star}\right) \delta_{x^{\star}}$ for $\alpha \in\{a, b\}$, where $c$ is a positive constant that will be fixed later and $F_{n}$ is the number of tiles in the patch that underlies $\mathcal{M}_{n}$, which is a level- $n$ Fibonacci number (with initial conditions $F_{0}=F_{1}=1$ in this case) and independent of the realisation of $\mathcal{M}_{n}$.

Note that the $\mu_{\alpha}^{(n)}$ are finite and positive pure point measures on the internal space $H=\mathbb{R}$, all with support in $W=[-\tau, \tau]$. Moreover, we work in a setting where $\frac{1}{c}\left(\mu_{a}^{(n)}+\mu_{b}^{(n)}\right)$ is a probability measure for each $n \in \mathbb{N}$. Now, we need a fundamental property of these measures, which is a reformulation of earlier results from [31,32, 33]. Let $g \cdot \mu:=\mu \circ g^{-1}$ denote the push-forward of a measure $\mu$ by a continuous (and, in our case, always invertible) function $g$. Define affine functions $g_{0}, f_{0}$ and $f_{1}$ by $g_{0}(x)=\sigma x+1$ and $f_{j}(x)=\sigma(x+j)$, where $\sigma:=\tau^{\star}=\tau^{\prime}=1-\tau$ is the algebraic conjugate of $\tau$. Then, an explicit calculation similar to the one from [32, Eq. 6.28] shows that we have the recursion

$$
\begin{aligned}
& \mu_{a}^{(n+1)}=\frac{F_{n}}{F_{n+1}}\left(p\left(g_{0} \cdot \mu_{a}^{(n)}\right)+q\left(f_{0} \cdot \mu_{a}^{(n)}\right)+\left(f_{0} \cdot \mu_{b}^{(n)}\right)\right), \\
& \mu_{b}^{(n+1)}=\frac{F_{n}}{F_{n+1}}\left(p\left(f_{0} \cdot \mu_{a}^{(n)}\right)+q\left(f_{1} \cdot \mu_{a}^{(n)}\right)\right),
\end{aligned}
$$

with initial condition $\mu_{a}^{(1)}=\delta_{0}$ and $\mu_{b}^{(1)}=0$.

Lemma 3.10. The sequences $\left(\mu_{\alpha}^{(n)}\right)_{n \in \mathbb{N}}$ of finite measures are weakly converging. The limit measures $\mu_{\alpha}$ are compactly supported and satisfy the system of rescaling equations

$$
\begin{aligned}
& \mu_{a}=|\sigma|\left(p\left(g_{0} \cdot \mu_{a}\right)+q\left(f_{0} \cdot \mu_{a}\right)+\left(f_{0} \cdot \mu_{b}\right)\right), \\
& \mu_{b}=|\sigma|\left(p\left(f_{0} \cdot \mu_{a}\right)+q\left(f_{1} \cdot \mu_{a}\right)\right),
\end{aligned}
$$

with $\sigma=\tau^{\prime}$ and with the functions $g_{0}$ and $f_{j}$ as in Eq. (14).

Proof. Since the support of any of the measures $\mu_{\alpha}^{(n)}$ is a finite subset of the compact set $W$, the claimed weak convergence follows from standard arguments, for instance by taking Fourier transforms and applying Levy's continuity theorem; see [15, Thm. 3.14]. Clearly, the support of the limit measure must also be contained in $W$.

With $F_{n} / F_{n+1} \stackrel{n \rightarrow \infty}{\longrightarrow} \tau^{-1}=|\sigma|$, it also follows that the limit measures satisfy the limiting rescaling relations, which are the ones stated.

Let us apply the Fourier transform to the relations of Lemma 3.10. Recall that the Fourier transform of a finite measure is a uniformly continuous function. Moreover, for an affine 
function $g$ defined by $g(x)=r x+s$ with $r \neq 0$, one obtains $\widehat{g . \mu}(t)=\mathrm{e}^{-2 \pi \text { ist }} \widehat{\mu}(r t)$; compare [32, Cor. 6.24]. This leads to the contractive relations

$$
\begin{aligned}
& \widehat{\mu_{a}}(t)=|\sigma|\left(\left(p \mathrm{e}^{-2 \pi \mathrm{i} t}+q\right) \widehat{\mu_{a}}(\sigma t)+\widehat{\mu_{b}}(\sigma t)\right), \\
& \widehat{\mu_{b}}(t)=|\sigma|\left(p+q \mathrm{e}^{-2 \pi \mathrm{i} \sigma t}\right) \widehat{\mu_{a}}(\sigma t) .
\end{aligned}
$$

One can check that a solution of this system, which is unique up to multiplication by a constant, is given by

$$
\begin{aligned}
& \widehat{\mu_{a}}(t)=\tilde{c} \mathrm{e}^{-\pi \mathrm{i} t} \operatorname{sinc}(\pi t) \prod_{\ell \geqslant 1}\left(p+q \mathrm{e}^{-2 \pi \mathrm{i} \sigma^{\ell} t}\right), \\
& \widehat{\mu_{b}}(t)=\tilde{c}|\sigma| \mathrm{e}^{-\pi \mathrm{i} \sigma t} \operatorname{sinc}(\pi \sigma t) \prod_{\ell \geqslant 1}\left(p+q \mathrm{e}^{-2 \pi \mathrm{i} \sigma^{\ell} t}\right),
\end{aligned}
$$

where the constant $\tilde{c}$ is determined by our additional condition that $\frac{1}{c}\left(\mu_{a}+\mu_{b}\right)$ is a probability measure, hence $\frac{1}{c}\left(\widehat{\mu_{a}}(0)+\widehat{\mu_{b}}(0)\right)=1$, which gives $\tilde{c}=c / \tau=c|\sigma|$. For reasons that will become clear below in Eq. (19), we choose $c=\tau$ and thus get $\tilde{c}=1$.

3.4. Continuity. As $\widehat{\mu_{a}}, \widehat{\mu_{b}} \in L^{2}(\mathbb{R})$, their (inverse) Fourier transforms are also elements of $L^{2}(\mathbb{R})$, and actually of $L^{2}(W)$, as they have compact support within $W$. But $L^{2}(W) \subset L^{1}(W)$ by Hölder's inequality, so the measures $\mu_{a}$ and $\mu_{b}$ are absolutely continuous with respect to Lebesgue measure $\lambda$, giving $\mu_{\alpha}=h_{\alpha} \lambda$ with Radon-Nikodym densities $h_{\alpha}$. By standard arguments, compare [39, Sec. 1.3.4] or [1, Thm. 2.2], one has

$$
\widehat{h_{a}}=\widehat{\mu_{a}} \quad \text { and } \quad \widehat{h_{b}}=\widehat{\mu_{b}},
$$

where $h_{\alpha}$ should not be thought of as a limit of the sequence $\left(h_{\alpha}^{(n)}\right)_{n \in \mathbb{N}}$ in the sense of functions. We will now show that the $L^{1}$-functions $h_{a}$ and $h_{b}$ are actually represented by continuous functions whenever $0<p<1$. To this end, we employ an old idea of Jessen and Wintner [26]. Let us first define

$$
\mu:=\underset{\ell=1}{\underset{*}{*}}\left(p \delta_{0}+q \delta_{\sigma^{\ell}}\right)=\underset{\ell=1}{\stackrel{\infty}{*}} \mu_{\ell},
$$

via the probability measures $\mu_{\ell}=p \delta_{0}+q \delta_{\sigma^{\ell}}$, where $p \in[0,1]$ and $q=1-p$. Note that the limiting cases are $\mu=\delta_{0}$ for $p=1$ and $\mu=\delta_{-\sigma^{2}}=\delta_{\tau-2}$ for $q=1$.

Lemma 3.11. The infinite convolution product for the measure $\mu$ from Eq. (16) is absolutely convergent to a probability measure in the weak topology, which is to say that it is weakly convergent to the same limit for any order of the terms.

Proof. With $M_{\kappa}(\nu):=\int_{\mathbb{R}} x^{\kappa} \mathrm{d} \nu(x)$ for $\kappa \geqslant 0$, we get $M_{0}\left(\mu_{\ell}\right)=1$ and $M_{\kappa}\left(\mu_{\ell}\right)=q \sigma^{\kappa \ell}$ for any $\kappa>0$. In particular, the second moments of all $\mu_{\ell}$ clearly exist. Moreover, one has

$$
\sum_{\ell=1}^{\infty}\left|M_{1}\left(\mu_{\ell}\right)\right|<\infty \text { and } \sum_{\ell=1}^{\infty} M_{2}\left(\mu_{\ell}\right)<\infty
$$

by a standard geometric series argument, because $|\sigma|=\tau^{-1}<1$. 
Hence, by an application of [26, Thm. 6], the convolution product is absolutely convergent as claimed. As all $\mu_{\ell}$ are probability measures, then so is the limit, $\mu$.

As a consequence, we may rewrite $\mu$ as $\mu=\mu_{j} * \nu_{j}$, for any $j \in \mathbb{N}$, where

$$
\nu_{j}:=\mu_{1} * \ldots * \mu_{j-1} * \mu_{j+1} * \ldots=\underset{i \neq j}{*} \mu_{i} .
$$

Together with the previous lemma, this has a rather strong consequence. In fact, the special case $p=\frac{1}{2}$ can be found in [26, Sec. 6].

Lemma 3.12. If $0<p<1$, the probability measure $\mu$ from Eq. (16) is continuous.

Proof. Observe first that, for any $j \in \mathbb{N}$ and with $\mu_{j}=p \delta_{0}+q \delta_{\sigma^{j}}$ as above, one has

$$
p q \mu_{j} \leqslant p \delta_{\sigma^{j}}+q \delta_{0} \leqslant p \delta_{\sigma^{j}}+q \delta_{2 \sigma^{j}}+q \delta_{0}+p \delta_{-\sigma^{j}}=\left(\delta_{\sigma^{j}}+\delta_{-\sigma^{j}}\right) * \mu_{j},
$$

which is to be understood as a relation between positive measures. The convolution with $\nu_{j}$ now leads to the general inequality

$$
p q \mu \leqslant\left(\delta_{\sigma^{j}}+\delta_{-\sigma^{j}}\right) * \mu .
$$

Assume now, to the contrary of our claim, that there is an element $x \in \mathbb{R}$ with $\mu(\{x\})>0$. Since $q=1-p$, we have $p, q \in(0,1)$ and thus $p q>0$. Then, Eq. (17) implies the estimate

$$
0<p q \mu(\{x\}) \leqslant \mu\left(\left\{x-\sigma^{j}\right\}\right)+\mu\left(\left\{x+\sigma^{j}\right\}\right) .
$$

Next, choose $r \in \mathbb{N}$ with $p q \mu(\{x\})>\frac{1}{r}$, which is clearly possible, and select $r$ distinct integers, $j_{1}<j_{2}<\ldots<j_{r}$, say. Since $\mu$ is a probability measure, we obtain

$$
\begin{aligned}
1 & \geqslant \mu\left(\bigcup_{1 \leqslant s \leqslant r}^{\dot{U}}\left(\left\{x-\sigma^{j_{s}}\right\} \dot{\cup}\left\{x+\sigma^{j_{s}}\right\}\right)\right) \\
& =\sum_{s=1}^{r}\left(\mu\left(\left\{x-\sigma^{j_{s}}\right\}\right)+\mu\left(\left\{x+\sigma^{j_{s}}\right\}\right)\right) \\
& \stackrel{(18)}{\geqslant} \sum_{s=1}^{r} p q \mu(\{x\})>r \frac{1}{r}=1 .
\end{aligned}
$$

This contradiction shows that $\mu$ is continuous.

Remark 3.13. Let us mention that one can also show $\mu$ to be a purely singular continuous measure. However, since we only need the continuity of $\mu$, we omit this extra step.

Before we continue, let us state a classic result that, due to the lack of a simple reference, we include here with a short proof.

Fact 3.14. Let $a, b \in \mathbb{R}$ with $a<b$, and let $\nu$ be a finite, regular Borel measure on $\mathbb{R}$ that is continuous, i.e., $\nu(\{x\})=0$ for all $x \in \mathbb{R}$. Now, let $J$ be any of the intervals $[a, b],(a, b)$, $[a, b)$ or $(a, b]$. Then, the function $1_{J} * \nu$ is continuous on $\mathbb{R}$. 
Proof. Consider the case of a closed interval first. Fix $x \in \mathbb{R}$ and choose a sequence $\left(x_{n}\right)_{n \in \mathbb{N}}$ with $x_{n} \searrow x$ as $n \rightarrow \infty$. Then, as $\nu$ is a continuous measure by assumption, we get

$$
\begin{aligned}
\left|\left(1_{J} * \nu\right)(x)-\left(1_{J} * \nu\right)\left(x_{n}\right)\right| & =\left|\int_{\mathbb{R}}\left(1_{J}(x-y)-1_{J}\left(x_{n}-y\right)\right) \mathrm{d} \nu(y)\right| \\
& =\left|\nu\left(\left[x-b, x_{n}-b\right]\right)-\nu\left(\left[x-a, x_{n}-a\right]\right)\right| \stackrel{n \rightarrow \infty}{\longrightarrow} 0
\end{aligned}
$$

because $\nu(\{x-b\})=0=\nu(\{x-a\})$. The analogous relation, with the same limit, holds when $x_{n} \nearrow x$. Together, this implies that the function $1_{J} * \nu$ is continuous on $\mathbb{R}$.

Due to the assumed continuity of $\nu$, the same type of argument applies for half-open or open intervals as well.

Proposition 3.15. If $0<p<1$, the Radon-Nikodym densities $h_{a}$ and $h_{b}$ are continuous functions with compact support.

Proof. Observe first that $\widehat{\mu}(t)=\prod_{\ell \geqslant 1}\left(p+q \mathrm{e}^{-2 \pi \mathrm{i} \sigma^{\ell} t}\right)$ by an application of the convolution theorem. Then, it follows from Eq. (15) that the $L^{1}$-functions $h_{a}$ and $h_{b}$ are represented by

$$
h_{a}(x)=\left(1_{[0,1)} * \mu\right)(x) \quad \text { and } \quad h_{b}(x)=\left(1_{[\sigma, 0)} * \mu\right)(x),
$$

with the probability measure $\mu$ from Eq. (16). Here, we have taken the liberty to choose halfopen intervals for the characteristic functions to match the standard situation in the limiting cases $p=1$ and $q=1$; compare the discussion in [4, Ex. 7.3].

Fact 3.14 in conjunction with Lemma 3.12 then implies the continuity of $h_{a}$ and $h_{b}$ for any $0<p<1$. Moreover, as a consequence of Lemma 3.10, both functions are supported on a subset of $W$.

Remark 3.16. Let us note that Eq. (19) also holds for the limiting cases $p=1$ and $q=1$, where one obtains $h_{a}=1_{[0,1)}$ and $h_{b}=1_{[1-\tau, 0)}$ as well as $h_{a}=1_{[\tau-2, \tau-1)}$ and $h_{b}=1_{[-1, \tau-2)}$, respectively. This is in line with the description of these deterministic limiting cases as regular model sets; compare [4, Rem. 4.6 and Ex. 7.3].

Now, for all $x \in \Lambda_{\geqslant 0}$, we need to relate the function values $h_{\alpha}\left(x^{\star}\right)$ with the occupation probabilities $g_{\alpha}(x)$ from above, because the continuity of the $h_{\alpha}$ is a representation result in the Lebesgue sense, but $\left(\Lambda_{\geqslant 0}\right)^{\star}$ is a null set.

Lemma 3.17. If $0<p<1$, one has $g_{\alpha}(x)=h_{\alpha}\left(x^{\star}\right)$ for $\alpha \in\{a, b\}$ and all $x \in \Lambda_{\geqslant 0}$.

Proof. When $0<p<1$, the functions $h_{\alpha}$ are continuous, and satisfy the recursions

$$
\begin{aligned}
& h_{a}\left(x^{\star}\right)=q h_{a}\left(\left(\frac{x}{\tau}\right)^{\star}\right)+h_{b}\left(\left(\frac{x}{\tau}\right)^{\star}\right)+p h_{a}\left(\left(\frac{x-1}{\tau}\right)^{\star}\right), \\
& h_{b}\left(x^{\star}\right)=p h_{a}\left(\left(\frac{x}{\tau}\right)^{\star}\right)+q h_{a}\left(\left(\frac{x-\tau}{\tau}\right)^{\star}\right),
\end{aligned}
$$

as a consequence of Lemma 3.10, rewritten in terms of the densities. With the initial condition $h_{a}\left(0^{\star}\right)=1 /(1+p)$, we see that we obtain the same type of renormalisation equation as in Eq. (13), and an application of Proposition 3.9 implies our claim. 
3.5. Diffraction measure of the random Fibonacci hull. Let us finally come back to the measure $\widehat{\gamma_{1}}$ from Eq. (8). We know from above that

$$
\widehat{\gamma_{1}}=\lim _{n \rightarrow \infty} \frac{1}{\tau^{n}}\left|\mathbb{E}\left(\mathcal{X}_{n}\right)\right|^{2}=\lim _{n \rightarrow \infty} \frac{1}{\tau^{n}}\left|\widehat{\mathbb{E}\left(\mathcal{M}_{n}\right)}\right|^{2}=\lim _{n \rightarrow \infty} \frac{1}{\tau^{n}} \mathcal{F}\left[\mathbb{E}\left(\mathcal{M}_{n}\right) * \widehat{\mathbb{E}\left(\mathcal{M}_{n}\right)}\right]
$$

where $\mathcal{F}$ denotes Fourier transform. By construction, $\mathbb{E}(\mathcal{M}):=\lim _{n \rightarrow \infty} \mathbb{E}\left(\mathcal{M}_{n}\right)$ is a weighted Dirac comb on $\mathcal{\curlywedge}([-\tau, \tau]) \cap \mathbb{R}_{\geqslant 0}$, with weights $g(x)=h\left(x^{\star}\right)$ and the interpretation given previously. With the second part of Proposition 3.9, one can now check that

$$
\widehat{\gamma_{1}}=\mathcal{F}(\mathbb{E}(\mathcal{M}) \circledast \widetilde{\mathbb{E}(\mathcal{M})}) \text {, }
$$

with $\circledast$ denoting the volume-averaged or Eberlein convolution of measures on $\mathbb{R}_{\geqslant 0}$. This holds as a consequence of the measure $|\mathbb{E}(\mathcal{M})|_{\left[0, L_{n}\right)}-\mathbb{E}\left(\mathcal{M}_{n}\right)\left|=\sum_{x \in \Lambda^{(n)}}\right| g(x)-g^{(n)}(x) \mid \delta_{x}$ getting uniformly small on $\Lambda^{(n)}$ as $n \rightarrow \infty$. In fact, one even has $\max _{x \in \Lambda^{(n)}}\left|g(x)-g^{(n)}(x)\right| \leqslant r^{n}$ with $r=\max \{p, q\}<1$. This implies, as in our earlier case, that

$$
\lim _{n \rightarrow \infty} \max _{x \in \Lambda^{(n)}}\left|g(x)-g^{(n)}(x)\right|=0,
$$

this time with exponentially fast convergence; compare [42, Lemma 3.29].

Next, define the two-sided measure

$$
\omega_{h}=\sum_{x \in \mathcal{\curlywedge}([-\tau, \tau])} h\left(x^{\star}\right) \delta_{x},
$$

with $h=h_{a}+h_{b}$ as before. It is not difficult to check that one then has

$$
\widehat{\gamma_{1}}=\widehat{\omega_{h} \circledast \widetilde{\omega}_{h}}=\widehat{\omega_{h * h}}
$$

where $\circledast$ now denotes the Eberlein convolution for measures on $\mathbb{R}$; see [4, Sec. 8.8]. Since we have $h_{a}, h_{b} \in C_{\mathrm{c}}(\mathbb{R})$ by Proposition 3.15, we may now apply the following general result.

Theorem 3.18 ([4, Thm. 9.5]). Consider the weighted Dirac comb

$$
\omega_{g}=\sum_{x \in \Lambda} g\left(x^{\star}\right) \delta_{x}
$$

on a regular model set $\Lambda=\curlywedge(W)$ with $C P S\left(\mathbb{R}^{d}, H, \mathcal{L}\right)$ and compact window $W=\overline{W^{\circ}} \subseteq H$, with a function $g: H \longrightarrow \mathbb{C}$ which is continuous on $W$ and vanishes on its complement. Then, $\omega_{g}$ has the positive, translation bounded, pure point diffraction measure

$$
\widehat{\gamma_{\omega_{g}}}=\sum_{k \in L^{\circledast}}|A(k)|^{2} \delta_{k} \quad \text { with } \quad A(k)=\operatorname{dens}(\mathcal{L}) \widehat{g}\left(-k^{\star}\right),
$$

where $L^{\circledast}=\pi\left(\mathcal{L}^{*}\right)$, with $\mathcal{L}^{*}$ the annihilator (or dual lattice) of $\mathcal{L}$, is the corresponding Fourier module.

It follows that $\widehat{\gamma_{1}}$ is pure point, with Fourier module $L^{\circledast}=\mathbb{Z}[\tau] / \sqrt{5}$ and amplitudes

$$
A_{\Lambda, p}(k)=\operatorname{dens}(\mathcal{L}) \widehat{h}\left(-k^{\star}\right)=\operatorname{dens}(\Lambda) \mathrm{e}^{\pi \mathrm{i}(1+\sigma) k^{\star}} \operatorname{sinc}\left(\pi(1-\sigma) k^{\star}\right) \prod_{\ell=1}^{\infty}\left(p+q \mathrm{e}^{2 \pi \mathrm{i} \sigma^{\ell} k^{\star}}\right),
$$


hence $\widehat{\gamma_{1}}=\sum_{k \in L^{\circledast}} I_{p}(k) \delta_{k}$ with $I_{p}(k)=\left|A_{\Lambda, p}(k)\right|^{2}$, so

$$
I_{p}(k)=\left(\frac{\tau}{\sqrt{5}} \operatorname{sinc}\left(\pi \tau k^{\star}\right)\right)^{2} \prod_{\ell=1}^{\infty}\left|p+q \mathrm{e}^{2 \pi \mathrm{i} \sigma^{\ell} k^{\star}}\right|^{2}=I(k) \prod_{\ell=1}^{\infty}\left|p+q \mathrm{e}^{2 \pi \mathrm{i} \sigma^{\ell} k^{\star}}\right|^{2} .
$$

Here, $I(k)$ is the intensity function from the deterministic case of Theorem 2.1 for $u_{a}=u_{b}=1$. Summarising the above derivations, we obtain the following result. Due to the mentioned compatibility of the measures on $\mathbb{Y}_{\zeta_{\mathrm{F}}}$ and on the subset $Y_{\zeta_{\mathrm{F}}}$, we may formulate it right away for the entire dynamical system $\left(\mathbb{Y}_{\zeta_{\mathrm{F}}}, \mathbb{R}, \nu_{\mathrm{pf}}\right)$.

Theorem 3.19. Fix some $\mathcal{T} \in \mathbb{Y}_{\zeta_{\mathrm{F}}}$ and let $\Lambda=\Lambda_{a} \dot{\cup} \Lambda_{b}$ be the corresponding set of left endpoints of the tiles in $\mathcal{T}$. Then, almost surely with respect to the ergodic patch frequency measure $\nu_{\mathrm{pf}}$, the corresponding diffraction measure reads

$$
\widehat{\gamma}=\mathbb{E}\left(\widehat{\gamma_{\Lambda}}\right)=\widehat{\gamma}_{\mathrm{pp}}+\widehat{\gamma}_{\mathrm{ac}}=\sum_{k \in \mathbb{Z}[\tau] / \sqrt{5}} I_{p}(k) \delta_{k}+\phi_{p} \lambda
$$

where $I_{p}(k)$ is given by Eq. (21) and $\phi_{p}$ is the Radon-Nikodym density of $\widehat{\gamma}_{a c}$, as computed previously in [32, Prop. 6.18].

Remark 3.20. The original formula for $\phi_{p}$ in $[32,33]$, which also appears in [22], can be made more explicit. Setting $\psi(k)=1-\cos \left(2 \pi \frac{k}{\tau}\right)$, one finds

$$
\phi_{p}(k)=\frac{2 p q \tau}{\sqrt{5}} \sum_{n=2}^{\infty} \frac{\psi(k)}{\tau^{n}} \prod_{\ell=1}^{n-2}\left|p+q \mathrm{e}^{-2 \pi \mathrm{i} \tau^{\ell} k}\right|^{2},
$$

with the understanding that an empty product is 1 . It is worthwhile to note that $\phi_{p}$ vanishes for the limiting cases $p=1$ and $q=1$, where $p q=0$. This means that the formula for $\widehat{\gamma}$ in Theorem 3.19 remains valid, with the correct result, for all values $p \in[0,1]$.

One can also extend the explicit formulas to the case of arbitrary weights $u_{a}, u_{b} \in \mathbb{C}$ for the two types of points. The intensities of the Bragg peaks are then the special case $m=1$ of the formula in Theorem 4.4 below, while the above formula for $\phi_{p}$ remains true, this time with $\psi(k)=\frac{1}{2}\left|\left(1-\mathrm{e}^{-2 \pi \mathrm{i} k}\right) u_{a}-\left(1-\mathrm{e}^{-2 \pi \mathrm{i} \tau k}\right) u_{b}\right|^{2}$.

One should notice that it is not immediately obvious why the supporting set of the Bragg peaks in the stochastic case coincides with that of the deterministic one. However, this ultimately follows from the fact that the deterministic substitutions $\zeta_{\mathrm{F}, 1}$ and $\zeta_{\mathrm{F}, 0}$ from Section 2 give rise to the same hull, and that the latter can be described within the same CPS as used there. This CPS then also accommodates the generating random Fibonacci sets, as described above. An independent explicit argument for the Fourier-Bohr spectrum via exponential sums can be found in [41]; see also [43] for a general treatment in the setting of almost periodic pure point measures. 
Remark 3.21. As before, we may also consider tiles with the modified lengths according to Eq. (4). In this case, one obtains

$$
\begin{gathered}
A_{\Lambda, p}^{\prime}(k)=A_{\Lambda}^{\prime}(k) \prod_{\ell=1}^{\infty}\left(p+q \mathrm{e}^{2 \pi \mathrm{i} \sigma^{\ell}\left(k^{\star}-\rho k\right)}\right) \quad \text { and } \\
I_{p}^{\prime}(k)=I^{\prime}(k) \prod_{\ell=1}^{\infty}\left|p+q \mathrm{e}^{2 \pi \mathrm{i} \sigma^{\ell}\left(k^{\star}-\rho k\right)}\right|^{2}
\end{gathered}
$$

as generalisations of the previous expressions, which are covered for $\rho=0$.

Also, the density function $\phi_{p}$ can be calculated for the case with the modified interval lengths. With the new length function $L_{\ell}=\tau^{\ell}+\rho \sigma^{\ell}$ for the level- $\ell$ inflation words, one finds

$$
\phi_{p}(k)=\frac{2 p q \tau}{\sqrt{5}} \sum_{n=2}^{\infty} \frac{\psi(k)}{\tau^{n}} \prod_{\ell=1}^{n-2}\left|p+q \mathrm{e}^{-2 \pi \mathrm{i} L_{\ell} k}\right|^{2},
$$

now with $\psi(k)=1-\cos (2 \pi(\rho \tau+\sigma) k)$.

\section{RANDOM NOBle MEANS SUbStitutions}

Let us begin this section with a more general approach to the concept of a random substitution; see [40] for some general properties and results.

Definition 4.1. Let a finite alphabet $\mathcal{A}=\left\{a_{1}, a_{2}, \ldots, a_{n}\right\}$ be fixed. Then, an endomorphism $\varrho: \mathcal{A}^{*} \longrightarrow \mathcal{A}^{*}$ is called a random substitution if there are $k_{1}, \ldots, k_{n} \in \mathbb{N}$ and probability vectors

$$
\left\{\boldsymbol{p}_{i}=\left(p_{i 1}, \ldots, p_{i k_{i}}\right) \mid \boldsymbol{p}_{i} \in[0,1]^{k_{i}} \text { and } \sum_{j=1}^{k_{i}} p_{i j}=1,1 \leqslant i \leqslant n\right\}
$$

such that

$$
\varrho: a_{i} \mapsto\left\{\begin{array}{cc}
w^{(i, 1)}, & \text { with probability } p_{i 1}, \\
\vdots & \vdots \\
w^{\left(i, k_{i}\right)}, & \text { with probability } p_{i k_{i}},
\end{array}\right.
$$

for $1 \leqslant i \leqslant n$, where each $w^{(i, j)} \in \mathcal{A}^{*}$. Moreover, the average

$$
M_{\varrho}:=\left(\sum_{q=1}^{k_{j}} p_{j q} \operatorname{card}_{a_{i}}\left(w^{(j, q)}\right)\right)_{1 \leqslant i, j \leqslant n} \in \operatorname{Mat}\left(n, \mathbb{R}_{\geqslant 0}\right)
$$

serves as the corresponding substitution matrix.

Remark 4.2. In principle, the integers $k_{i}$ may take the value $\infty$, but we do not consider such cases here. As in the deterministic case, a random substitution $\varrho$ is primitive if and only if $M_{\varrho}$ is a primitive matrix. Various other notions can also be extended; compare [40].

From a general point of view, each $\varrho\left(a_{i}\right)$ is a random variable, which means that our $M_{\varrho}$ is actually the expectation of the substitution matrix, the latter also viewed as a random 
variable. We suppress such extensions, as we do not need them for our systems of compatible substitutions.

The random Fibonacci substitution can be generalised as follows; compare [33] and references therein. Consider $\mathcal{A}=\{a, b\}$ as before, pick $m \in \mathbb{N}$ and let $\boldsymbol{p}_{m}=\left(p_{0}, \ldots, p_{m}\right)$ be a fixed probability vector. We shall usually assume that all $p_{i}>0$ unless specified otherwise. Define the deterministic substitutions $\zeta_{m, i}$ by $b \mapsto a \mapsto a^{i} b a^{m-i}$, for $0 \leqslant i \leqslant m$. These $m+1$ substitutions all define the same hull, and share the substitution matrix

$$
M^{(m)}=\left(\begin{array}{cc}
m & 1 \\
1 & 0
\end{array}\right)
$$

with PF eigenvalue $\lambda_{m}=\frac{1}{2}\left(m+\sqrt{m^{2}+4}\right)$, which is a Pisot-Vijayaraghavan (PV) number and a unit. Its algebraic conjugate, $\lambda_{m}^{\prime}=\frac{1}{2}\left(m-\sqrt{m^{2}+4}\right)$, is the other eigenvalue of $M^{(m)}$. For the geometric realisation as tilings with natural tile lengths, we choose $\lambda_{m}$ and 1 for the tiles (intervals) of type $a$ and $b$, respectively. One then obtains the analogue of Theorem 2.1, this time with the Fourier module

$$
\mathcal{F}_{m}=\frac{\mathbb{Z}\left[\lambda_{m}\right]}{\sqrt{m^{2}+4}}
$$

which covers our previous case for $m=1$.

Also, the description of $\Lambda=\Lambda_{a} \dot{\cup} \Lambda_{b}$ as model sets is completely analogous. Here, the lattice is $\mathcal{L}_{m}=\left\{\left(x, x^{\star}\right) \mid x \in \mathbb{Z}\left[\lambda_{m}\right]\right\}$, where $x^{\star}=x^{\prime}$ is algebraic conjugation in the quadratic field $\mathbb{Q}\left(\lambda_{m}\right)=\mathbb{Q}\left(\sqrt{m^{2}+4}\right)$. It turns out (see $[4,32]$ for background and details) that the windows for $\zeta_{m, j}$ with $0 \leqslant j \leqslant m$ may be chosen as

$$
W_{m, j}^{(a)}=j \tau_{m}+[0,1) \quad \text { and } \quad W_{m, j}^{(b)}=j \tau_{m}+\left[\lambda_{m}^{\prime}, 0\right)
$$

with $\tau_{m}=\frac{-1}{m}\left(\lambda_{m}^{\prime}+1\right)$. The choice with the half-open intervals is only relevant for $j=0$ and $j=m$, where the fixed points are singular (as in the Fibonacci case); compare [4, Ex. 7.3] for a discussion of this point. Since the hull defined by $\zeta_{m, j}$ is independent of $j$, we get the following result; see [38, 4] for background.

Corollary 4.3. Fix $m \in \mathbb{N}$ and let $\mathbb{Y}_{m}$ be the geometric hull of the corresponding noble means tiling system, with prototiles of length $\lambda_{m}$ for type a and 1 for type $b$. Fix some $\mathcal{T} \in \mathbb{Y}_{m}$ and let $\Lambda=\Lambda_{a} \dot{\cup} \Lambda_{b}$ be the corresponding set of left endpoints of the tiles in $\mathcal{T}$. Then, the weighted Dirac comb $\omega=u_{a} \delta_{\Lambda_{a}}+u_{b} \delta_{\Lambda_{b}}$, with any fixed pair of weights $u_{a}, u_{b} \in \mathbb{C}$, is pure point diffractive. Its autocorrelation is given by

$$
\gamma=\sum_{z \in \Lambda-\Lambda} \sum_{\alpha, \beta \in\{a, b\}} \overline{u_{\alpha}} \eta_{\alpha \beta}(z) u_{\beta} \delta_{z}
$$

where $\eta_{\alpha \beta}(z)=\operatorname{dens}\left(\Lambda_{\alpha} \cap\left(\Lambda_{\beta}-z\right)\right)$, and the diffraction measure reads $\widehat{\gamma}=\sum_{k \in \mathcal{F}_{m}} I(k) \delta_{k}$, where $\mathcal{F}_{m}$ is the Fourier module from Eq. (22). As before, $I(k)=\left|u_{a} A_{\Lambda_{a}}(k)+u_{b} A_{\Lambda_{b}}(k)\right|^{2}$, with the Fourier-Bohr coefficients from Theorem 2.1. In particular, $\gamma$ and $\Lambda-\Lambda$, as well as $\widehat{\gamma}$ and $I(k)$, are independent of $\mathcal{T}$. 
Now, given $m \in \mathbb{N}$, the random noble mean substitution $\zeta_{m}: \mathcal{A}^{*} \longrightarrow \mathcal{A}^{*}$ is defined by

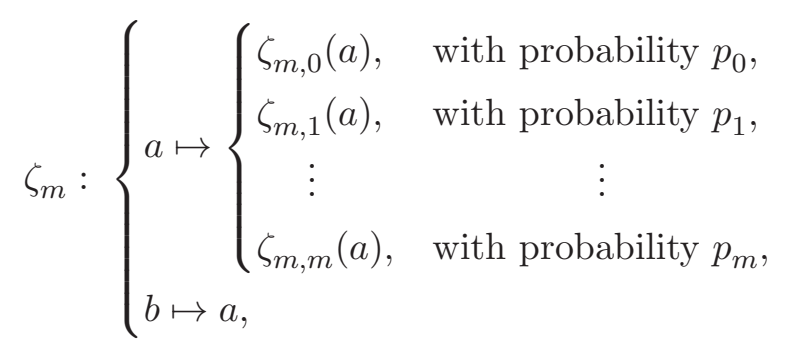

and the one-parameter family $\mathcal{R}=\left\{\zeta_{m}\right\}_{m \in \mathbb{N}}$ is called the family of random noble means substitutions (RNMS). In particular, one has $\zeta_{1}=\zeta_{\mathrm{F}}$.

From here on, we can continue in close analogy to the Fibonacci case. Eq. (9) is now to be replaced by $m+1$ equations. Explicitly, one has

$$
\mathcal{M}_{n}=\sum_{r=0}^{j-1}\left(\delta_{r \lambda_{m}^{n-1}} * \mathcal{M}_{n-1}^{(r)}\right)+\delta_{j \lambda_{m}^{n-1}} * \mathcal{M}_{n-2}+\sum_{r=j}^{m-1}\left(\delta_{\lambda_{m}^{n-2}+r \lambda_{m}^{n-1}} * \mathcal{M}_{n-1}^{(r)}\right)
$$

with probability $p_{j}$, for $0 \leqslant j \leqslant m$. Here, empty sums are 0 as usual, and $\mathcal{M}_{n-1}^{(0)}, \ldots, \mathcal{M}_{n-1}^{(m-1)}$ are $m$ independent and identically distributed copies of the random variable $\mathcal{M}_{n-1}$, which is an important point to observe in comparison to the previous case, $m=1$.

In this case, one gets $\mathbb{E}\left(\mathcal{M}_{n}\right)=\sum_{x \in \mathcal{\lambda}\left(W_{m}\right) \cap \mathbb{R}_{\geqslant 0}} h^{(n)}\left(x^{\star}\right) \delta_{x}$, now with covering window $W_{m}=\left[\lambda_{m}^{\prime}-1,1-\lambda_{m}^{\prime}\right]$ and model set $\curlywedge\left(W_{m}\right)=\left\{x \in \mathbb{Z}\left[\lambda_{m}\right] \mid x^{\star} \in W_{m}\right\}$. As before, one has $h^{(n)}=h_{a}^{(n)}+h_{b}^{(n)}$, and with the analogous definition of the measures $\mu_{\alpha}^{(n)}$ one arrives again at a set of recursion relations. They can be used to establish the existence of the limiting measures $\mu_{\alpha}$, which then satisfy the rescaling relations

$$
\begin{aligned}
& \mu_{a}=\left|\lambda_{m}^{\prime}\right| \sum_{n=0}^{m} p_{n}\left(\sum_{j=0}^{n-1}\left(f_{j} \cdot \mu_{a}\right)+\sum_{j=n}^{m-1}\left(g_{j} \cdot \mu_{a}\right)+\left(f_{0} \cdot \mu_{b}\right)\right), \\
& \mu_{b}=\left|\lambda_{m}^{\prime}\right| \sum_{n=0}^{m} p_{n}\left(f_{n} \cdot \mu_{a}\right),
\end{aligned}
$$

where $f_{j}(x)=\lambda_{m}^{\prime}(x+j)$ and $g_{j}(x)=\lambda_{m}^{\prime}(x+j)+1$, while empty sums are 0 by convention. The solutions are again absolutely continuous measures. They can be represented by $\mu_{a}=h_{a} \lambda$ and $\mu_{b}=h_{b} \lambda$ with

$$
h_{a}(x)=\left(1_{[0,1)} * \mu\right)(x) \text { and } h_{b}(x)=\left(1_{\left[\lambda_{m}^{\prime}, 0\right)} * \mu\right)(x),
$$

where we now have

$$
\mu=\underset{\ell=1}{*}\left(\sum_{n=0}^{m} p_{n} \delta_{n\left(\lambda_{m}^{\prime}\right)^{\ell}}\right) .
$$

When $\boldsymbol{p}$ is strictly positive, the $L^{1}$-functions $h_{a}$ and $h_{b}$ are again represented by continuous functions with compact support (by an analogous argument), hence Theorem 3.18 can be applied. This gives the following result. 
Theorem 4.4. Let $m \in \mathbb{N}$ be fixed and consider the random noble means substitution from Eq. (23). Let $\mathbb{Y}_{\zeta}$ be the geometric tiling hull, with intervals of length $\lambda_{m}$ and 1 as prototiles, and consider the dynamical system $\left(\mathbb{Y}_{\zeta}, \mathbb{R}, \nu_{\mathrm{pf}}\right)$, with the ergodic patch frequency measure $\nu_{\mathrm{pf}}$.

Then, for $\nu_{\mathrm{pf}}$-almost every element $\mathcal{T} \in \mathbb{Y}_{\zeta}$, with $\Lambda=\Lambda_{a} \dot{\cup} \Lambda_{b}$ denoting the left endpoints of $\mathcal{T}$, the diffraction measure of the weighted Dirac comb $\omega=u_{a} \delta_{\Lambda_{a}}+u_{b} \delta_{\Lambda_{b}}$ is of the form

$$
\widehat{\gamma_{\Lambda}}=\widehat{\gamma}_{\mathrm{pp}}+\widehat{\gamma}_{\mathrm{ac}}=\sum_{k \in \mathcal{F}_{m}} I_{\boldsymbol{p}}(k) \delta_{k}+\phi_{\boldsymbol{p}} \lambda
$$

with the Fourier module $\mathcal{F}_{m}=\mathbb{Z}\left[\lambda_{m}\right] / \sqrt{m^{2}+4}$. The Bragg peak intensities are given by

$$
I_{\boldsymbol{p}}(k)=I(k) \prod_{\ell=1}^{\infty}\left|\sum_{n=0}^{m} p_{n} \mathrm{e}^{2 \pi \mathrm{i} n\left(\lambda_{m}^{\prime}\right)^{\ell} k^{\star}}\right|^{2},
$$

where $I(k)=\left|\operatorname{dens}\left(\Lambda_{m}\right) \operatorname{sinc}\left(\pi\left(1-\lambda_{m}^{\prime}\right) k^{\star}\right)\right|^{2}$ with $\operatorname{dens}\left(\Lambda_{m}\right)=\frac{1-\lambda_{m}^{\prime}}{\sqrt{m^{2}+4}}$ is the 'deterministic' part. Finally, the Radon-Nikodym density of the absolutely continuous part reads

$$
\phi_{\boldsymbol{p}}(k)=\frac{\lambda_{m}}{\sqrt{m^{2}+4}} \sum_{\ell=2}^{\infty} \frac{\psi_{\boldsymbol{p}}^{(\ell)}(k)}{\lambda_{m}^{\ell}},
$$

where $\psi_{\boldsymbol{p}}^{(\ell)}$ are uniformly bounded, continuous functions on $\mathbb{R}$.

Sketch of proof. As indicated, Theorem 3.18 covers the case that $\boldsymbol{p}$ is strictly positive. It can then be shown that any of the remaining limiting cases where some of the $p_{i}$ vanish is still covered by the same formula. Consequently, the result holds for all probability vectors and recovers the model set case with its pure point diffraction in the deterministic limits, where we have $p_{i}=1$ for a single index, in which case $\phi_{\boldsymbol{p}}$ vanishes.

Modified tile lengths can also be considered. Since the resulting changes are structurally similar to those encountered in the special case $m=1$, we omit further details, some of which will be presented in [42].

\section{Deterministic PERIOd DOUbLing CHAin}

In the next section, we are going to investigate a locally randomised version of the period doubling substitution. Therefore, let us first recall what is known about the deterministic substitution, which has constant length and is defined by

$$
\rho_{\mathrm{pd}}: \quad a \mapsto a b, \quad b \mapsto a a ;
$$

see [4, Sec. 4.5.1, Ex. 7.4 and Sec. 9.4.4] for background. In analogy to Theorem 2.1, one obtains the following result; compare [38, Ch. V] and [4, Sec. 9.4.4].

Theorem 5.1. Let $\mathbb{Y}_{\mathrm{pd}}$ be the geometric hull of the period doubling tiling system, with two distinct prototiles of length 1 , and $\mathbb{Y}_{0}=\left\{y \in \mathbb{Y}_{\mathrm{pd}} \mid 0 \in y\right\}$ its discrete counterpart. Then, the topological dynamical systems $\left(\mathbb{Y}_{0}, \mathbb{Z}\right)$ and $\left(\mathbb{Y}_{\mathrm{pd}}, \mathbb{R}\right)$ are strictly ergodic, both with pure point dynamical spectrum. 
Now, fix some $\mathcal{T} \in \mathbb{Y}_{\mathrm{pd}}$ and let $\Lambda=\Lambda_{a} \dot{\cup} \Lambda_{b}$ be the corresponding set of left endpoints of the tiles in $\mathcal{T}$. Then, the weighted Dirac comb $\omega=u_{a} \delta_{\Lambda_{a}}+u_{b} \delta_{\Lambda_{b}}$ with any fixed pair of weights $u_{a}, u_{b} \in \mathbb{C}$ is pure point diffractive. Its autocorrelation $\gamma$ can be expressed as in Theorem 2.1, while the diffraction measure reads

$$
\widehat{\gamma}=\sum_{k \in \mathbb{Z}\left[\frac{1}{2}\right]} I(k) \delta_{k},
$$

with $I(k)=\left|u_{a} A_{\Lambda_{a}}(k)+u_{b} A_{\Lambda_{b}}(k)\right|^{2}$ and Fourier-Bohr coefficients defined as in Theorem 2.1. In particular, $\gamma$ and $\Lambda-\Lambda$, as well as $\widehat{\gamma}$ and $I(k)$, are independent of $\mathcal{T}$, while the FourierBohr coefficients do depend on the chosen element, but converge uniformly.

Again, the elements of $\mathbb{Y}_{\text {pd }}$ can be understood as (translates of) regular model sets. For this purpose, choose $H=\mathbb{Z}_{2}$, the set of 2-adic integers, as locally compact Abelian group to obtain a $\operatorname{CPS}\left(\mathbb{R}, \mathbb{Z}_{2}, \mathcal{L}\right)$ with lattice

$$
\mathcal{L}=\{(x, \iota(x)) \mid x \in \mathbb{Z}\} \subset \mathbb{R} \times \mathbb{Z}_{2},
$$

where $\iota: \mathbb{Z} \hookrightarrow \mathbb{Z}_{2}$ is the canonical embedding, which is also the $\star$-map in this case. In particular, one can describe the fixed point under $\rho_{\text {pd }}^{2}$ with seed $a \mid a$ as a regular model set in this way; see [11] as well as [4, Ex. 7.4] for details.

It is well known that the diffraction measure $\widehat{\gamma_{\mathrm{pd}}}$ of the corresponding Dirac comb, which is also the diffraction measure of the entire system $\mathbb{Y}_{\mathrm{pd}}$, is pure point. For generic choices of the weights $u_{a}$ and $u_{b}$, the set of Bragg peak positions is a group, namely

$$
L^{\circledast}=\mathbb{Z}\left[\frac{1}{2}\right]=\left\{\frac{m}{2^{r}} \mid(r=0, m \in \mathbb{Z}) \text { or }(r \geqslant 1, m \text { odd })\right\},
$$

which means that there are then no extinctions. Let us mention in passing that $L^{\circledast}$ is also the dynamical spectrum for the dynamical system under the continuous translation action of $\mathbb{R}$, while the restriction of $L^{\circledast}$ to the 1 -torus $\mathbb{T}$, here written as $[0,1)$ with addition modulo 1 , is the spectrum for the discrete $\mathbb{Z}$-action by the shift.

In our parametrisation, with $k=\frac{m}{2^{r}}$, the Fourier-Bohr coefficients (or amplitudes) of our particular Dirac comb are given by

$$
A_{\Lambda_{a}}(k)=\frac{2}{3} \frac{(-1)^{r}}{2^{r}} \mathrm{e}^{2 \pi \mathrm{i} k} \quad \text { and } \quad A_{\Lambda_{b}}(k)=\delta_{r, 0}-A_{\Lambda_{a}}(k) .
$$

Hence, the diffraction intensities for $k \in L^{\circledast}$ can be calculated as

$$
I(k)= \begin{cases}\frac{1}{9 \cdot 4^{r-1}}\left|u_{a}-u_{b}\right|^{2}, & r \geqslant 1, \\ \frac{1}{9}\left|2 u_{a}+u_{b}\right|^{2}, & r=0 .\end{cases}
$$

Let us note in passing that $u_{a}=u_{b}$ leads to $I(k)=0$ for all $k=\frac{m}{2^{r}}$ with $m$ odd and $r \geqslant 1$ because the Dirac comb then 'degenerates' to $u_{a} \delta_{\mathbb{Z}}$, wherefore $\widehat{\gamma}$ simply becomes $\left|u_{a}\right|^{2} \delta_{\mathbb{Z}}$ and is 'blind' to the aperiodicity that is present for $u_{a} \neq u_{b}$.

Now, let us consider the alternative substitution

$$
\rho_{\mathrm{pd}}^{\prime}: \quad a \mapsto b a, b \mapsto a a,
$$


which is conjugate to $\rho_{\text {pd }}$ by an inner automorphism of the free group with generators $a$ and b. By [4, Prop. 4.6.], the substitutions $\rho_{\text {pd }}$ and $\rho_{\text {pd }}^{\prime}$ define the same two-sided hull. Due to the constant-length nature of these substitutions, the symbolic and the geometric picture coincide canonically, for instance by choosing (coloured) intervals of unit length as prototiles (as we did above). In particular, we can then identify the hulls $\mathbb{X}_{\mathrm{pd}}$ and $\mathbb{Y}_{0}$. Moreover, the relation between the topological dynamical systems $\left(\mathbb{Y}_{0}, \mathbb{Z}\right)$ and $\left(\mathbb{Y}_{\mathrm{pd}}, \mathbb{R}\right)$ is given by a simple suspension with constant roof function; see [16] for background. We shall see more of this in Section 7.4.

Remark 5.2. The analysis of the system under a change of the tile lengths is considerably more involved here in comparison to the Fibonacci case. This is due to the nature of $\mathbb{Z}_{2}$ as internal space. In particular, it is no longer true that changing the tile length ratio leads to a deformed model set. This can also be seen as a consequence of topological obstructions identified in [17].

Clearly, global mixtures of $\rho_{\mathrm{pd}}$ and $\rho_{\mathrm{pd}}^{\prime}$ do not lead to an extension of the hull, and are thus compatible in this sense, as in our previous examples. Once again, this situation changes under local mixtures, as we shall see next.

\section{RANDOM PERIOD DOUBLING CHAIN}

Now, fix $p \in[0,1]$, set $q=1-p$, and define the random period doubling substitution by

$$
\rho:\left\{\begin{array}{l}
a \mapsto \begin{cases}a b, & \text { with probability } p, \\
b a, & \text { with probability } q,\end{cases} \\
b \mapsto a a,
\end{array}\right.
$$

which has substitution matrix $M=\left(\begin{array}{ll}1 & 2 \\ 1 & 0\end{array}\right)$, independently of $p$. As before, the term 'random' is only justified for $p \in(0,1)$, while the limiting cases correspond to the deterministic cases of the previous section. Again, for $p \in(0,1)$, we define the two-sided discrete stochastic hull $\mathbb{X}_{\rho}$ as

$$
\mathbb{X}_{\rho}:=\left\{w \in \mathcal{A}^{\mathbb{Z}} \mid \mathfrak{F}(\{w\}) \subseteq \mathcal{D}_{\rho}\right\},
$$

with the notation from Definition 3.1. It is clear by construction that $\mathbb{X}_{\rho}$ contains the deterministic hull as a proper subset.

6.1. Entropy. For the stochastic hull, one has the following result.

Lemma 6.1. For $p \in(0,1)$, the topological entropy of $\left(\mathbb{X}_{\rho}, \mathbb{Z}\right)$ is $s=\frac{2}{3} \log (2) \approx 0.462$.

Proof. As long as $0<p<1$, the dictionary of legal words is always the same, and the hull contains elements with dense orbits under the $\mathbb{Z}$-action of the shift. Any element of such an orbit contains all finite legal words, wherefore the topological entropy $s$ equals the patch counting entropy of such an element; compare [25]. Consequently, if $\mathcal{W}_{n}$ is the set of legal words of length $n$, one has $s=\lim _{n \rightarrow \infty} \frac{1}{n} \log \left|\mathcal{W}_{n}\right|$, where $|\cdot|$ denotes the cardinality of a set. 
Note that the sequence $\left(\left|\mathcal{W}_{n}\right|\right)_{n \in \mathbb{N}}$ is subadditive, wherefore the limit exists by Fekete's lemma $[20]$.

Consider the pedigree graph of successive exact substitution words that originate from $a$ as its seed (or level 0). Due to the constant-length nature of the random period doubling substitution, this graph has the property that the words on any given level (defined by graph distance from $a$ ) are distinct. In other words, the graph is a tree, with root $a$. This tree contains words of length $2^{r}$ for any $r \in \mathbb{N}_{0}$, but only a subset of $\mathcal{W}_{2^{r}}$ on level $r$, for any $r \in \mathbb{N}_{0}$.

It is easy to check inductively that all exact substitution words of length $2^{r}$ in this tree contain $\frac{1}{3}\left(2^{r+1}+(-1)^{r}\right)$ letters $a$. Moreover, since each $a$ (multiplicatively) gives rise to two distinct words on the next level, the total number $\#_{r}$ of exact substitution words of length $2^{r}$, by induction, is given by

$$
\#_{r}=2^{\left(2^{r+2}-(-1)^{r}-3\right) / 6},
$$

with $\lim _{r \rightarrow \infty} 2^{-r} \log \left(\#_{r}\right)=\frac{2}{3} \log (2)$, so that this clearly is a lower bound for $s$.

Now, consider a legal word of length $2 n$, with $n \geqslant 2$ say. This word must either emerge as the substitution of a legal word of length $n$, or of one of length $n-1$, then completed with a prefix and a suffix of one letter each. If $m_{n}$ denotes the maximal number of $a$ 's in any element of $\mathcal{W}_{n}$, we thus have the estimate

$$
\left|\mathcal{W}_{2 n}\right| \leqslant 2^{m_{n}}\left|\mathcal{W}_{n}\right|+4 \cdot 2^{m_{n-1}}\left|\mathcal{W}_{n-1}\right|<5 \cdot 2^{m_{n}}\left|\mathcal{W}_{n}\right|
$$

which implies $s \leqslant \log (2) \liminf _{n \rightarrow \infty} \frac{m_{n}}{n}$ by standard arguments. Since $\liminf _{n \rightarrow \infty} \frac{m_{n}}{n}$ is bounded from above by $\frac{2}{3}$, which is the frequency of $a$ 's according to standard PerronFrobenius theory with the substitution matrix $M$, our lower bound for $s$ is also its upper bound, and the claim on the entropy follows.

Remark 6.2. Let us note that the last part of the proof of Lemma 6.1 actually also shows that $\liminf _{n \rightarrow \infty} \frac{m_{n}}{n}=\frac{2}{3}$ and hence

$$
\lim _{n \rightarrow \infty} \frac{m_{n}}{n}=\frac{2}{3}
$$

This follows from the observation that $\limsup _{n \rightarrow \infty} \frac{m_{n}}{n}=\frac{2}{3}+\varepsilon$ with $\varepsilon>0$ would imply the existence of a sequence of words $\left(w_{n_{i}}\right)_{i \in \mathbb{N}}$ with the frequency of $a$ 's converging to $\frac{2}{3}+\varepsilon$. But then, $\left(\rho_{\text {pd }}\left(w_{n_{i}}\right)\right)_{i \in \mathbb{N}}$ would define another sequence of words with the frequencies of $a$ 's converging to $\frac{2}{3}-\frac{\varepsilon}{2}<\frac{2}{3}$, which is impossible.

An analogous argument shows that the minimal number of $a$ 's in the legal words of length $n$ asymptotically grows like $\frac{2}{3} n$ as well, and not slower, so that each element of our stochastic hull $\mathbb{X}_{\rho}$ has well-defined frequencies $\frac{2}{3}$ and $\frac{1}{3}$ for the letters $a$ and $b$, respectively.

6.2. Diffraction. The diffraction measure is given by the obvious modification of Eq. (6) to this case, where we need to consider distinct weights for the two types of points. We want to proceed by the same method as before. Therefore, using the corresponding concatenation 
rule, we obtain

$$
\mathcal{X}_{n}(k)= \begin{cases}\mathcal{X}_{n-1}(k)+\mathrm{e}^{-2 \pi \mathrm{i} k \cdot 2^{n-1}} \mathcal{X}_{n-2}(k)+\mathrm{e}^{-2 \pi \mathrm{i} k \cdot 3 \cdot 2^{n-2}} \mathcal{X}_{n-2}^{\prime}(k), & \text { with prob. } p, \\ \mathcal{X}_{n-2}(k)+\mathrm{e}^{-2 \pi \mathrm{i} k \cdot 2^{n-2}} \mathcal{X}_{n-2}^{\prime}(k)+\mathrm{e}^{-2 \pi \mathrm{i} k \cdot 2^{n-1}} \mathcal{X}_{n-1}(k), & \text { with prob. } q,\end{cases}
$$

together with $\mathcal{X}_{0}(k)=u_{a}$ and

$$
\mathcal{X}_{1}(k)= \begin{cases}u_{a}+u_{b} \mathrm{e}^{-2 \pi \mathrm{i} k}, & \text { with probability } p, \\ u_{b}+u_{a} \mathrm{e}^{-2 \pi \mathrm{i} k}, & \text { with probability } q .\end{cases}
$$

Note that, in Eq. (27), $\mathcal{X}_{n-2}$ and $\mathcal{X}_{n-2}^{\prime}$ are independent random variables with the same distribution, as in our previous RNMS case.

As before, $\left(\mathcal{X}_{n}(k)\right)_{n \in \mathbb{N}}$ is a subsequence of $\left(X_{m}(k)\right)_{m \in \mathbb{N}}$, and we obtain

$$
\mathbb{E}\left(\widehat{\gamma_{\Lambda}}\right)=\lim _{n \rightarrow \infty} \frac{1}{2^{n}}\left|\mathbb{E}\left(\mathcal{X}_{n}\right)\right|^{2}+\lim _{n \rightarrow \infty} \frac{1}{2^{n}} \operatorname{Var}\left(\mathcal{X}_{n}\right)=: \widehat{\gamma_{1}}+\widehat{\gamma_{2}}
$$

We have

$$
\widehat{\gamma_{1}}=\lim _{n \rightarrow \infty} \frac{1}{2^{n}}\left|\widehat{\mathbb{E}\left(\mathcal{M}_{n}\right)}\right|^{2}=\lim _{n \rightarrow \infty} \frac{1}{2^{n}} \mathcal{F}\left[\mathbb{E}\left(\mathcal{M}_{n}\right) * \widehat{\mathbb{E}\left(\mathcal{M}_{n}\right)}\right]
$$

where $\mathcal{M}_{n}$ is the measure with $\widehat{\mathcal{M}_{n}}=\mathcal{X}_{n}$. By construction, $\mathbb{E}(\mathcal{M})=\lim _{n \rightarrow \infty} \mathbb{E}\left(\mathcal{M}_{n}\right)$ is a weighted Dirac comb on $\mathbb{N}_{0}$, where the weight at $x \in \mathbb{N}_{0}$ is given by

$$
u_{a} \mathbb{P}(\text { type at } x \text { is } a)+u_{b} \mathbb{P}(\text { type at } x \text { is } b) .
$$

In analogy to our treatment in Section 3, one now finds

$$
\widehat{\gamma_{1}}=\mathcal{F}[\mathbb{E}(\mathcal{M}) \circledast \widetilde{\mathbb{E}(\mathcal{M})}],
$$

where we tacitly assume that the volume-averaging for $\circledast$ is taken with the appropriate weights for one-sided sequences. The underlying reason is that the positive pure point measure $|\mathbb{E}(\mathcal{M})|_{\left[0,2^{n}\right)}-\mathbb{E}\left(\mathcal{M}_{n}\right) \mid$ gets uniformly small on $\mathbb{Z} \cap\left[0,2^{n}\right)$ as $n \rightarrow \infty$; see [42] for an explicit derivation of this fact, and the Appendix for a general approach that gives the result we need from a weaker assumption.

6.3. Averages and weight function. Next, we observe that we get

$$
\mathbb{E}(\mathcal{M})=u_{b} \delta_{\mathbb{N}_{0}}+\left(u_{a}-u_{b}\right) \sum_{x \in \mathbb{N}_{0}} a_{x} \delta_{x}
$$

with $a_{x}=\mathbb{P}$ (type at $x$ is $\left.a\right)$. Thus, we can restrict our attention to the case $u_{a}=1$ and $u_{b}=0$, which reduces Eq. (28) to $\mathbb{E}(\mathcal{M})=\sum_{x \in \mathbb{N}_{0}} a_{x} \delta_{x}$. It is not difficult to employ the random substitution to derive the following recursive structure of the probabilities $a_{x}$.

Fact 6.3. For any $n \in \mathbb{N}_{0}$, one has

$$
a_{2 n}=1-q a_{n} \quad \text { and } \quad a_{2 n+1}=1-p a_{n} .
$$

In particular, this gives $a_{0}=\frac{1}{1+q}$. 
Next, we aim at defining a function $h: \mathbb{Z}_{2} \longrightarrow[0,1]$ in analogy to our previous approach. We begin by setting $h(n)=a_{n}$ for any $n \in \mathbb{N}_{0}$, where we canonically identify $\mathbb{N}_{0}$ with its image $\iota\left(\mathbb{N}_{0}\right)$ in the 2 -adic integers. To extend $h$, we will use a uniform continuity argument, for which we first need an intermediate result.

Lemma 6.4. For arbitrary $m, j, k \in \mathbb{N}_{0}$, one has

$$
\begin{aligned}
h\left(m 2^{j}\right) & =h(0)+(h(m)-h(0))(-q)^{j} \quad \text { and } \\
h\left(m 2^{j}+k\right) & =h(k)+x_{j}, \quad \text { with }\left|x_{j}\right| \leqslant(\max \{p, q\})^{j} .
\end{aligned}
$$

Proof. The first identity follows from a simple inductive calculation (in $j$ ) with the recursion from Fact 6.3 for $h\left(m 2^{j}\right)=a_{m 2^{j}}$.

The second property can be shown by induction in $k$. For $k=0$, and any $m, j \in \mathbb{N}_{0}$, the claim follows from the first identity, because $|h(m)-h(0)|$ is bounded by 1 . Now, let the assertion be true (for arbitrary $m, j \in \mathbb{N}_{0}$ ) for $1, \ldots, k$, where we first look at the case that $k=2 r$ is even. Then, with $r \leqslant k$, one gets

$$
\begin{aligned}
h\left(m 2^{j}+k+1\right) & =h\left(2\left(m 2^{j-1}+r\right)+1\right)=1-p h\left(m 2^{j-1}+r\right) \\
& =1-p\left(h(r)+x_{j-1}\right)=1-p h(r)-p x_{j-1}=h(k+1)-x_{j},
\end{aligned}
$$

where $\left|x_{j}\right| \leqslant \max \{p, q\}\left|x_{j-1}\right|$. The case $k$ odd can be handled analogously.

As before, in order to apply Theorem 3.18, the next aim is to extend $h$ to a continuous function on all of $\mathbb{Z}_{2}$ such that $a_{n}=h\left(n^{\star}\right)=h(\iota(n))$. We will see that it is enough to consider the dense subset $\mathbb{N}_{0} \subset \mathbb{Z}_{2}$, where the denseness of $\mathbb{N}_{0}$ is a consequence of that of $\mathbb{Z}$ together with the identity $1+2+4+\ldots=-1$ in $\mathbb{Z}_{2}$. For $n \in \mathbb{N}_{0}$, we have $h\left(n^{\star}\right)=h(n)$ because the $\star$-map is the identity on $\mathbb{Z}$, due to our canonical identification of $\mathbb{Z}$ with $\iota(\mathbb{Z})$.

Lemma 6.5. The function $h$ can be extended to a uniformly continuous function on $\mathbb{Z}_{2}$.

Proof. Let $\varepsilon>0$ be fixed, choose $j \in \mathbb{N}$ such that $(\max \{p, q\})^{j}<\varepsilon$ and set $\delta:=2^{-j}$. Then, we have $|x-y|_{2} \leqslant \delta$ with $x, y \in \mathbb{N}_{0}$ if and only if $x-y$ is divisible by $2^{j}$, so $y=x+m 2^{j}$ for some $m \in \mathbb{N}_{0}$. Hence, by Lemma 6.4 , we obtain

$$
|f(x)-f(y)|=\left|f(x)-f\left(x+m 2^{j}\right)\right|=\left|x_{j}\right| \leqslant(\max \{p, q\})^{j}<\varepsilon .
$$

As $\mathbb{N}_{0} \subset \mathbb{Z}_{2}$ is dense and $h$ is uniformly continuous on $\mathbb{N}_{0}$, we know that $h$ can be extended to a uniformly continuous function on $\mathbb{Z}_{2}$; see [18, (3.15.6)]. By slight abuse of notation, this extension is still called $h$.

Remark 6.6. While the argument we used to prove the continuity of $h$ in this section looks different from our previous argument, the basic idea is the same. Indeed, consider a CPS $(G, H, \mathcal{L})$, and denote by $L=\pi(\mathcal{L}) \subset G$ the projection of the lattice. Then, the image $L^{\star}$ of $L$ under the star mapping is dense in $H$.

In the previous sections, we showed that the function $h$ is uniformly continuous by constructing it as an infinite convolution and showing the continuity of the convolution, which was made easy by the simple structure of the group $H=\mathbb{R}$. This approach would also work 
here, but the computations are more involved due to the 2-adic structure of $H$. What we did instead was to pull back $h$ through the star mapping to the function $g(x)=h\left(x^{\star}\right)$, and show that $g$ is uniformly continuous in the induced topology of the embedding $\star: L \hookrightarrow H$. Then, the 2-adic structure of $H$ makes the induced topology easy to work with, while the induced topology would be harder to tackle in the CPS of the previous sections.

6.4. Diffraction: Pure point part. With this preparation, we define the two-sided comb $\omega_{h}=\sum_{x \in \mathbb{Z}} h(x) \delta_{x}$, and we then have $\widehat{\gamma_{1}}=\left(\omega_{h} \circledast \widetilde{\omega_{h}}\right)^{\widehat{ }}=\widehat{\omega_{h * \widetilde{h}}}$ in complete analogy to our previous cases. This is once again a pure point measure, by another application of Theorem 3.18 .

Let us assume for a moment that $\widehat{\gamma_{2}}$ is a continuous measure (which we will prove below). In this case, we can apply a variant of [24, Thm. 3.2] and [30, Thm. 5 and Cor. 5] to obtain

$$
\mathbb{E}\left(\widehat{\gamma_{\Lambda}}\right)(\{k\})=\lim _{n \rightarrow \infty} \frac{1}{4^{n}}\left|\mathbb{E}\left(\mathcal{X}_{n}(k)\right)\right|^{2}
$$

From Eq. (27), setting $E_{n}:=\mathbb{E}\left(\mathcal{X}_{n}\right)$, we infer that

$$
E_{n}=\left(p+q \mathrm{e}^{-2^{n} \pi \mathrm{i} k}\right) E_{n-1}+\left(q+q \mathrm{e}^{-2^{n-1} \pi \mathrm{i} k}+p \mathrm{e}^{-2^{n} \pi \mathrm{i} k}+p \mathrm{e}^{-3 \cdot 2^{n-1} \pi \mathrm{i} k}\right) E_{n-2}
$$

together with $E_{0}(k)=1$ and $E_{1}(k)=p+q \mathrm{e}^{-2 \pi \mathrm{i} k}$. In particular, $E_{1}(\ell)=1$ for all $\ell \in \mathbb{Z}$. Recall that every $k \in L^{\circledast}=\mathbb{Z}\left[\frac{1}{2}\right]$ can be written in the form $k=\frac{m}{2^{r}}$. If $n \geqslant r+2$, one has

$$
E_{n}=E_{n-1}+2 E_{n-2} \text {. }
$$

With the initial conditions $E_{r}$ and $E_{r+1}$, this recurrence relation has the unique solution

$$
E_{n}=\frac{1}{3}\left(2^{n-r}\left(E_{r}+E_{r+1}\right)+(-1)^{n-r}\left(2 E_{r}-E_{r+1}\right)\right) .
$$

Combining this with Eq. (29), we obtain

$$
\mathbb{E}\left(\widehat{\gamma_{\Lambda}}\right)\left(\left\{\frac{m}{2^{r}}\right\}\right)=\frac{1}{9 \cdot 4^{r}}\left|E_{r}\left(\frac{m}{2^{r}}\right)+E_{r+1}\left(\frac{m}{2^{r}}\right)\right|^{2} .
$$

Applying Eq. (30), it is not difficult to see that $E_{r+1}\left(\frac{m}{2^{r}}\right)=2 E_{r}\left(\frac{m}{2^{r}}\right)$. Hence, we have

$$
\mathbb{E}\left(\widehat{\gamma_{\Lambda}}\right)\left(\left\{\frac{m}{2^{r}}\right\}\right)=\frac{1}{9 \cdot 4^{r-1}}\left|E_{r}\left(\frac{m}{2^{r}}\right)\right|^{2} .
$$

Moreover, if we apply Eq. (30) inductively, we get

$$
\begin{aligned}
E_{r}\left(\frac{m}{2^{r}}\right) & =\left(E_{r-1}\left(\frac{m}{2^{r}}\right)-\left(1+\mathrm{e}^{-2^{r-1} \pi \mathrm{i} \frac{m}{2^{r}}}\right) E_{r-2}\left(\frac{m}{2^{r}}\right)\right)\left(-q-p \mathrm{e}^{-2^{r} \pi \mathrm{i} \frac{m}{2^{r}}}\right) \\
& =\left(E_{1}\left(\frac{m}{2^{r}}\right)-\left(1+\mathrm{e}^{-2 \pi \mathrm{i} \frac{m}{2^{r}}}\right) E_{0}\left(\frac{m}{2^{r}}\right)\right) \prod_{\ell=2}^{r}\left(-q-p \mathrm{e}^{-2^{\ell} \pi \mathrm{i} \frac{m}{2^{r}}}\right) \\
& =\prod_{\ell=1}^{r}\left(-q-p \mathrm{e}^{-2^{\ell} \pi \mathrm{i} \frac{m}{2^{r}}}\right) .
\end{aligned}
$$

Finally, we arrive at

$$
\mathbb{E}\left(\widehat{\gamma_{\Lambda}}\right)\left(\left\{\frac{m}{2^{r}}\right\}\right)=\frac{1}{9 \cdot 4^{r-1}} \prod_{\ell=1}^{r}\left|q+p \mathrm{e}^{-2^{\ell} \pi \mathrm{i} \frac{m}{2^{r}}}\right|^{2}=\widehat{\gamma_{\Lambda, \operatorname{det}}}\left(\left\{\frac{m}{2^{r}}\right\}\right) \prod_{\ell=1}^{r}\left|q+p \mathrm{e}^{-2^{\ell} \pi \mathrm{i} \frac{m}{2^{r}}}\right|^{2} .
$$


Remark 6.7. As in the case of the random Fibonacci substitution, we find that the Bragg peak intensities in the stochastic situation are given by the deterministic ones multiplied by some function that depends on the probabilities $p$ and $q$. For example, one obtains

$$
\mathbb{E}\left(\widehat{\gamma_{\Lambda}}\right)\left(\left\{\frac{1}{2}\right\}\right)=(p-q)^{2} \widehat{\gamma_{\Lambda, \operatorname{det}}}\left(\left\{\frac{1}{2}\right\}\right),
$$

while $\widehat{\gamma_{\Lambda}}(\{0\})=\widehat{\gamma_{\Lambda, \operatorname{det}}}(\{0\})=\frac{4}{9}$, independently of $p$ and for every $\Lambda \in \mathbb{Y}_{\mathrm{pd}}$.

6.5. Diffraction: Continuous part. Let us now focus on $\widehat{\gamma_{2}}$. Following the same idea as in [32, Sec. 6.2.3], we obtain a recurrence relation for $V_{n}:=\operatorname{Var}\left(\mathcal{X}_{n}\right)$,

$$
V_{n}=V_{n-1}+2 V_{n-1}+2 p q \psi_{n}, \quad \text { for } n \geqslant 2,
$$

with $V_{0} \equiv 0$ and $V_{1}(k)=2 p q(1-\cos (2 \pi k))$, where the functions $\psi_{n}$ are defined by

$$
\psi_{n}(k):=\frac{1}{2}\left|\left(1-\mathrm{e}^{-2 \pi \mathrm{i} 2^{n-1} k}\right) E_{n-1}-\left(1+\mathrm{e}^{-2 \pi \mathrm{i} 2^{n-2} k}-\mathrm{e}^{-2 \pi \mathrm{i} 2^{n-1} k}-\mathrm{e}^{2 \pi \mathrm{i} 3 \cdot 2^{n-2} k}\right) E_{n-2}\right|^{2} .
$$

Via induction, the functions $\psi_{n}$ can be expressed more explicitly as follows.

Lemma 6.8. We have

$$
\psi_{n}(k)=\left(1-\cos \left(2^{n} \pi k\right)\right) \prod_{\ell=1}^{n-1}\left|q+p \mathrm{e}^{-2^{\ell} \pi \mathrm{i} k}\right|^{2} .
$$

In particular, we obtain $\psi_{n}(k) \leqslant 2$ for all $n \geqslant 2$.

This leads to the following observation.

Proposition 6.9. The measure $\widehat{\gamma_{2}}$ is absolutely continuous with respect to $\lambda$. Its RadonNikodym density is given by the continuous function

$$
\phi_{p}(k)=\frac{1}{3} V_{1}+\frac{4 p q}{3} \sum_{j=2}^{\infty} 2^{-j} \psi_{j}(k)=\frac{4 p q}{3} \sum_{n=1}^{\infty} \frac{1-\cos \left(2^{n} \pi k\right)}{2^{n}} \prod_{j=1}^{n-1}\left|q+p \mathrm{e}^{-2^{j} \pi \mathrm{i} k}\right|^{2} .
$$

Proof. Define $\alpha_{n}=\frac{1}{3}\left(2^{n}-(-1)^{n}\right)$. One can easily show via induction that

$$
V_{n}=\alpha_{n} V_{1}+2 p q \sum_{j=2}^{n} \alpha_{n+1-j} \psi_{j}
$$

which implies

$$
\frac{V_{n}}{2^{n}}=\frac{1}{3} \frac{2^{n}-(-1)^{n}}{2^{n}} V_{1}+2 p q \sum_{j=2}^{n} \frac{1}{3} \frac{2^{n+1-j}-(-1)^{n+1-j}}{2^{n}} \psi_{j}
$$

Now, it is not difficult to see that $\frac{V_{n}(k)}{2^{n}}$ converges uniformly to $\phi_{p}(k)$. The second equality then follows from Lemma 6.8.

If we collect our findings, we obtain the following result. 
Theorem 6.10. Fix some $\mathcal{T} \in \mathbb{Y}_{\rho}$ and let $\Lambda=\Lambda_{a} \dot{\cup} \Lambda_{b}$ be the corresponding set of left endpoints of the tiles in $\mathcal{T}$. Then, almost surely with respect to the patch frequency measure $\nu_{\mathrm{pf}}$ of the random period doubling substitution, the corresponding diffraction measure reads

$$
\widehat{\gamma}=\widehat{\gamma}_{\mathrm{pp}}+\widehat{\gamma}_{\mathrm{ac}}=\sum_{k \in \mathbb{Z}[1 / 2]} I_{p}(k) \delta_{k}+\phi_{p} \lambda,
$$

where $I_{p}(k)$ is given by Eq. (31) and $\phi_{p}$ is the Radon-Nikodym density of $\widehat{\gamma}_{\mathrm{ac}}$, as stated in Proposition 6.9.

\section{Eigenfunctions And Kronecker factor}

It is a common feature of the above examples that their pure point part could be determined in closed form, and deeply resembled the formulas known from weighted model sets, though the systems themselves do certainly not belong to this class. Moreover, in view of positive topological entropy and the structure of the hulls, one cannot expect all eigenfunctions to be continuous. Consequently, the maximal equicontinuous factor (MEF) will not be the right tool to proceed.

Instead, one has to identify the Kronecker factor, which emerges as the maximal pure point factor under a measurable map, where we are allowed to work up to a null set of the hull. In fact, this is where the covering model set will enter, and the well-defined MEF of this model set will be the Kronecker factor of our compatible random inflation systems.

7.1. Covers and eigenfunctions. To explain what happens, we will first discuss the random Fibonacci inflation from Section 3, which means that we consider the dynamical system $(\mathbb{Y}, \mathbb{R}, \nu)$ with $\mathbb{Y}=\mathbb{Y}_{\zeta_{\mathrm{F}}}$ and $\nu=\nu_{\mathrm{pf}}$. Recall that $a$ and $b$ stand for intervals of length $\tau$ and 1 , respectively, both with a reference point on their left end. Consider all possible infinite one-sided tilings (to the right of the origin, that is) that emerge as a realisation of an infinite inflation process from a single tile, $a$ say, with its reference point at 0 . In this situation, if we code a point by a pair $(\alpha, x)$ with $\alpha \in\{a, b\}$, one inflation step, on the level of individual points, means

$$
(b, x) \mapsto(a, \tau x) \quad \text { and } \quad(a, x) \mapsto \begin{cases}\{(b, \tau x),(a, \tau x+1)\}, & \text { with prob. } p, \\ \{(a, \tau x),(b, \tau x+\tau)\}, & \text { with prob. } q .\end{cases}
$$

Let us assume $p q \neq 0$, which excludes the two deterministic cases, and let $\Lambda_{\alpha}$ be the union of all type- $\alpha$ positions of all realisations, and consider the sets $W_{\alpha}:=\overline{\Lambda_{\alpha}}$ in internal space. It is clear that these sets must satisfy the system of equations given by

$$
\begin{aligned}
& W_{a}=\sigma W_{a} \cup \sigma W_{b} \cup\left(\sigma W_{a}+1\right), \\
& W_{b}=\sigma W_{a} \cup\left(\sigma W_{a}+\sigma\right),
\end{aligned}
$$

where $\sigma=\tau^{\prime}$ as before. Since $|\sigma|<1$, (32) defines a contractive iterated function system on $\mathcal{K} \times \mathcal{K}$, where $\mathcal{K}$ is the set of compact subsets of $\mathbb{R}$ equipped with the Hausdorff metric; see $[10,44]$ for background. 
Fact 7.1. The compact sets $W_{a}=[-1, \tau]$ and $W_{b}=[-\tau, 1 / \tau]$ are the unique solution to Eq. (32) within $\mathcal{K} \times \mathcal{K}$, and one has $W=W_{a} \cup W_{b}=[-\tau, \tau]$.

Proof. Since $\mathcal{K} \times \mathcal{K}$ with the Hausdorff metric is a complete metric space, and the iteration defined by the right-hand side of (32) is a contraction in this space, with contraction constant $|\sigma|$, Banach's contraction principle guarantees a unique fixed point, which then is the solution of (32). One can now check by a simple calculation that the intervals $W_{a}$ and $W_{b}$ as stated solve this equation.

One immediate consequence is that, for each possible realisation, the points of type $\alpha$ are a subset of the regular model set $\curlywedge\left(W_{\alpha}\right)$ in the CPS of the Fibonacci chain from Eq. (1). The question now is to what extent these windows are determined by a single realisation. For an answer, we employ the so-called 'chaos game' [37, Sec. 4.2] and Elton's ergodic theorem [19]; see also [13, Thm. 10] or [37, p. 127].

Consider the single-point iteration in internal space, as defined by $p(0)=(a, 0)$ together with $p(n+1)=\Theta(p(n))$ for $n \geqslant 0$, where $\Theta$ is a random mapping in internal space, defined by

$$
(b, y) \mapsto(a, \sigma y) \quad \text { and } \quad(a, y) \mapsto \begin{cases}(a, \sigma y+1), & \text { with prob. } p_{1}, \\ (a, \sigma y), & \text { with prob. } p_{2}, \\ (b, \sigma y), & \text { with prob. } p_{3}, \\ (b, \sigma y+\sigma), & \text { with prob. } p_{4},\end{cases}
$$

where $p_{i}>0$ and $\sum_{i} p_{i}=1$. Now, Elton's theorem asserts that, almost surely, the corresponding (infinite) random point sequences lie dense in the attractor of the IFS, as long as all $p_{i}>0$.

In direct space, each such sequence is an (exponentially thin) subset of a possible realisation, and the previous argument shows that already this thin subset, almost surely, has a dense lift into the two windows. This establishes the following result.

Proposition 7.2. Almost every realisation of the one-sided random Fibonacci inflation tiling completely determines the windows of the covering two-component model set, in the sense that the lift of the positions of type $\alpha$ via the $\star$-map lies dense in the compact set $W_{\alpha}$. In particular, the lift of all left endpoints together is a dense subset of $W=[-\tau, \tau]$.

The corresponding result applies to one-sided tilings that extend to the left, for instance when starting from $(b,-1)$ or from $(a,-\tau)$. By intersecting two sets of full measure, one obtains the following consequence.

Corollary 7.3. Almost every realisation of the two-sided random Fibonacci inflation tiling that emerges from one of the central seeds $a|a, a| b, b \mid a$ or $b \mid b$ completely determines the window of the covering model set, as in Proposition 7.2.

Let $Y_{0}$ denote the set (or fibre) of all two-sided realisations according to Corollary 7.3. Of course, there are realisations in $Y_{0}$ that do not fix the window, such as the ones that give 
perfect Fibonacci chains, but all such cases together are only a null set. Here, as mentioned earlier, the relevant measure on $Y_{0}$ is the one induced by $\nu$ on it via filtration, and agrees with the one defined by the inflation process according to Figure 1; see [23]. This also means that some elements of $Y_{0}$ are thinnings not just of one model set, but of many, and this applies analogously to all translates $Y_{t}=t+Y_{0}$. This is the origin of the discontinuity of non-trivial eigenfunctions, as we analyse next.

Recall that $0 \neq f \in L^{2}(\mathbb{Y}, \nu)$ is called an eigenfunction of $(\mathbb{Y}, \mathbb{R}, \nu)$ if there exists a $k \in \mathbb{R}$ such that

$$
f(t+y)=\mathrm{e}^{2 \pi \mathrm{i} k t} f(y)
$$

holds for all $t \in \mathbb{R}$ and $\nu$-a.e. $y \in \mathbb{Y}$. Moreover, $f$ is called continuous if there is a continuous function on $\mathbb{Y}$ such that (33) holds for all $y \in \mathbb{Y}$. In this case, $k$ is called a topological eigenvalue (in additive notation).

Proposition 7.4. The topological point spectrum of $(\mathbb{Y}, \mathbb{R}, \nu)$ is trivial, which is to say that the only continuous eigenfunction is the constant one.

Proof. Let $f$ be a continuous eigenfunction. Since $(\mathbb{Y}, \mathbb{R}, \nu)$ is ergodic, $|f|$ is a constant, which we may choose to be 1 . Let $y \in Y_{0}$ be fixed, and set $c=f(y)$. Due to the structure of the fibre $Y_{0}$, the tiling $y$ is of the form $y_{\mathrm{L}} \mid y_{\mathrm{R}}$, which is to say that it consists of two infinite half-tilings that are glued together at 0 . Let $y^{\prime} \in Y_{0}$ be any other element, which is then of the form $y_{\mathrm{L}}^{\prime} \mid y_{\mathrm{R}}^{\prime}$. Now, $Y_{0}$ clearly also contains the element $y_{\mathrm{L}} \mid y_{\mathrm{R}}^{\prime}$, and one has

$$
f\left(y^{\prime}\right)=f\left(y_{\mathrm{L}}^{\prime} \mid y_{\mathrm{R}}^{\prime}\right)=f\left(y_{\mathrm{L}} \mid y_{\mathrm{R}}^{\prime}\right)=f\left(y_{\mathrm{L}} \mid y_{\mathrm{R}}\right)=f(y)=c,
$$

because continuous eigenfunctions cannot distinguish between two right-asymptotic or between two left-asymptotic elements. Consequently, $f$ is constant on $Y_{0}$.

Now, consider the two inflation tilings that correspond to the two fixed points of the square of the Fibonacci inflation $b \mapsto a \mapsto a b$, with seeds $a \mid a$ and $b \mid a$, called $y_{1}$ and $y_{2}$. By construction, both are elements of $Y_{0}$. At the same time, the left endpoints (of the tiles of types $a$ and $b$ ) are regular model sets, given by

$$
\boldsymbol{八}^{(a)}([\tau-2, \tau-1)), \boldsymbol{\curlywedge}^{(b)}([-1, \tau-2)) \text { and } \quad \boldsymbol{八}^{(a)}((\tau-2, \tau-1]), \boldsymbol{八}^{(b)}((-1, \tau-2]),
$$

respectively. A comparison with the windows for $\Lambda_{a}$ and $\Lambda_{b}$ now shows that also $\tau+y_{1}$ and $1+y_{2}$ are in $Y_{0}$, wherefore we may conclude that $f\left(y_{1}\right)=f\left(\tau+y_{1}\right)=\mathrm{e}^{2 \pi \mathrm{i} \tau k} f\left(y_{1}\right)$ and $f\left(y_{2}\right)=f\left(1+y_{2}\right)=\mathrm{e}^{2 \pi \mathrm{i} k} f\left(y_{2}\right)$. This implies $k=0$ and $f$ is thus the constant function as claimed.

This shows why we cannot work with the MEF of $(\mathbb{Y}, \mathbb{R}, \nu)$, which is trivial, but need to consider its Kronecker factor instead. 
7.2. Torus parametrisation and Kronecker factor. It will be instrumental to employ the MEF of another dynamical system as follows. Let $\Lambda=\curlywedge(W)$ with $W=[-\tau, \tau]$ be the covering model set from above, in the CPS $(\mathbb{R}, H, \mathcal{L})$ from $(1)$, where we use $H=\mathbb{R}$ to explicitly distinguish direct and internal space in our following arguments. It follows from the standard theory of model sets via dynamical systems [8] that $\Lambda$ defines a strictly ergodic dynamical system that is a.e. one to one over its MEF. The latter is $\mathbb{A}=(\mathbb{R} \times H) / \mathcal{L}$ together with the induced translation action of $\mathbb{R}$ on it. Here, $\mathbb{A}$ is a 2-torus, and a translation by $t \in \mathbb{R}$ is represented as a translation by $(t, 0)$ modulo $\mathcal{L}$ on $\mathbb{A}$. Moreover, we also have the classic torus parametrisation at hand, where we assume that $\Lambda$, which is a singular element, is the union of all elements in the fibre over $(0,0) \in \mathbb{A}$.

The connection now works as follows. The fibre $Y_{0}$ is linked to $\Lambda$ itself and hence mapped to $(0,0)$. Since a.e. element in the fibre determines the window of $\Lambda$ uniquely by Corollary 7.3 , we can unambiguously map these elements to $(0,0)$. To extend this to a mapping from $\nu$-a.e. element of $\mathbb{Y}$ to $\mathbb{A}$, we first select a generic element $y_{0} \in Y_{0}$. Now, for any $y \in \mathbb{Y}$, there is a sequence $\left(t_{n}\right)_{n \in \mathbb{N}}$ of translations such that

$$
y=\lim _{n \rightarrow \infty}\left(t_{n}+y_{0}\right) .
$$

It clearly suffices to consider the transversal of $\mathbb{Y}$, which is to say that we may assume $y \in \mathbb{Y}_{0}:=\{u \in \mathbb{Y} \mid 0 \in u\}$ without loss of generality. The advantage is that we now always have $y \subset \mathbb{Z}[\tau]$, so all $t_{n}$ in (34) lie in $\mathbb{Z}[\tau]$ as well, and the $\star$-map is well defined. Also, the convergence then simply means that we may choose $t_{n}$ such that $y \cap[-n, n]=\left(t_{n}+y_{0}\right) \cap[-n, n]$ holds, because our point sets have finite local complexity.

Lemma 7.5. If $(r, s)$ is a cluster point of $\left(t_{n}, 0\right)_{n \in \mathbb{N}}$ in $\mathbb{A}$, with the translations $t_{n}$ from Eq. (34), we have $-r+y \subseteq \curlywedge(-s+W)$.

Proof. Let $U$ and $V$ be open, relatively compact neighbourhoods of $0 \in \mathbb{R}$ and $0 \in H$, respectively, and assume $V=-V$. Then, our assumption implies that there is a subsequence $\left(n_{j}\right)_{j \in \mathbb{N}}$ of integers such that

$$
\left(t_{n_{j}}, 0\right) \in(r, s)+U \times V+\mathcal{L}
$$

holds for all sufficiently large $j$, say $j>N$. For any such $j$, we have $n_{j}>j$ and thus

$$
y \cap[-j, j]=\left(t_{n_{j}}+y_{0}\right) \cap[-j, j] \subseteq t_{n_{j}}+y_{0} \subseteq t_{n_{j}}+\curlywedge(W) .
$$

By $(35)$, we have $\left(t_{n_{j}}, 0\right)=(r, s)+(u, v)+\left(x, x^{\star}\right)$ for some $u \in U, v \in V$ and $\left(x, x^{\star}\right) \in \mathcal{L}$, hence $t_{n_{j}}=r+u+x$ and $s+v+x^{\star}=0$. Consequently, we have $y \cap[-j, j] \subseteq r+u+x+人(W)$, where $x+\curlywedge(W)=\curlywedge\left(x^{\star}+W\right)$ because $\left(x, x^{\star}\right) \in \mathcal{L}$. This implies

$y \cap[-j, j] \subseteq r+u+\curlywedge(-s-v+W) \subseteq r+U+\curlywedge(-s+W-V)=r+U+\curlywedge(-s+W+V)$,

which holds for all $j>N$ and thus implies

$$
y=\bigcup_{j>N} y \cap[-j, j] \subseteq r+U+\curlywedge(-s+W+V) .
$$


Since this holds for any open neighbourhood $U$ of 0 , and since $\curlywedge(-s+W+V)$ is a Delone set due to the relative compactness of $V$, we get

$$
\bigcap_{0 \in U \text { open }} U+(r+\curlywedge(-s+W+V))=r+\curlywedge(-s+W+V)
$$

so that $y \subseteq r+\curlywedge(-s+W+V)$ and hence also

$$
y \subseteq \bigcap_{\substack{0 \in V=-V \\ V \text { open }}} r+\curlywedge(-s+W+V) \supseteq r+\curlywedge(-s+W) .
$$

Now, our claim follows if we show that the last inclusion actually is an equality.

To do so, we may assume $r=0$ without loss of generality. Let $x \in L \backslash \curlywedge(-s+W)$, where $L=\pi(\mathcal{L})$ from the CPS $(1)$, so $x^{\star} \notin-s+W$. Then, there is an open neighbourhood $V$ of $0 \in H$ with $V=-V$ such that $\left(x^{\star}+V\right) \cap(-s+W)=\varnothing$, which implies that $x^{\star} \notin-s+W+V$ and thus $x \notin \curlywedge(-s+W+V)$. Consequently, $y \in r+\curlywedge(-s+W)$ as claimed.

Next, in order to define a proper mapping from (a subset of) $\mathbb{Y}$ to the torus, we need to get rid of the subsequences from the previous lemma.

Corollary 7.6. If $y \in \mathbb{Y}$ is generic, there exists a unique $(r, s) \in \mathbb{A}$ such that

$$
-r+y \subseteq \curlywedge(-s+W) .
$$

Proof. Since $\mathbb{A}$ is compact, any sequence $\left(t_{n}, 0\right)_{n \in \mathbb{N}}$ in $\mathbb{A}$ has at least one cluster point. Therefore, Lemma 7.5 gives the existence, and it remains to show uniqueness.

Let $-r_{i}+y \subseteq \curlywedge\left(-s_{i}+W\right)$ for $i \in\{1,2\}$, hence also the inclusions $\left(-r_{i}+y\right)^{\star} \subseteq-s_{i}+W$ and thus $s_{i}+\left(-r_{i}+y\right)^{\star} \subseteq W$, which means that the sets $-r_{i}+y$ are translates of elements in our special fibre, $Y_{0}$. When $y$ is generic (in the measure-theoretic sense), the window is uniquely determined, which is to say that

$$
\overline{s_{i}+\left(-r_{i}+y\right)^{\star}}=s_{i}+\overline{\left(-r_{i}+y\right)^{\star}}=W, \quad \text { for } i \in\{1,2\} .
$$

But this implies

$$
\begin{aligned}
-s_{2}+W & =\overline{\left(-r_{2}+y\right)^{\star}}=\overline{\left(-r_{2}+r_{1}-r_{1}+y\right)^{\star}} \\
& =\left(r_{1}-r_{2}\right)^{\star}+\overline{\left(-r_{1}+y\right)^{\star}}=\left(r_{1}-r_{2}\right)^{\star}-s_{1}+W .
\end{aligned}
$$

Since $v+W=W$ is only possible for $v=0$, we conclude that $s_{1}-s_{2}=\left(r_{1}-r_{2}\right)^{\star}$, which means nothing but $\left(r_{1}-r_{2}, s_{1}-s_{2}\right) \in \mathcal{L}$ and our claim follows.

At this point, we can define

$$
\mathbb{Y}^{\prime}:=\{y \in \mathbb{Y}: \text { there is a unique }(r, s) \in \mathbb{A} \text { with }-r+y \subseteq \curlywedge(-s+W)\},
$$

and we then have a well-defined mapping $\psi: \mathbb{Y}^{\prime} \longrightarrow \mathbb{A}$. By Corollary 7.6, $\mathbb{Y}^{\prime}$ contains all generic elements and thus has full measure.

Proposition 7.7. The mapping $\psi: \mathbb{Y}^{\prime} \longrightarrow \mathbb{A}$ is continuous. 
Proof. Since $\mathbb{R}$ is metrisable, the same property holds for $\mathbb{Y}$ and $\mathbb{A}$, and we may work with sequences. Let $y_{n} \in \mathbb{Y}^{\prime}$ with $n \in \mathbb{N}$ be chosen so that $y_{n} \longrightarrow y$ in $\mathbb{Y}^{\prime}$ as $n \rightarrow \infty$. We then need to show that $\psi\left(y_{n}\right) \longrightarrow \psi(y)$.

Let $\psi\left(y_{n}\right)=\left(r_{n}, s_{n}\right)$ and $\psi(y)=(r, s)$. Since $\mathbb{A}$ is compact, it suffices to show that any cluster point of $\left(r_{n}, s_{n}\right)$ equals $(r, s)$ modulo $\mathcal{L}$. Let $\left(r^{\prime}, s^{\prime}\right)$ be a cluster point of the sequence, so $\left(r_{k_{n}}, s_{k_{n}}\right) \longrightarrow\left(r^{\prime}, s^{\prime}\right)$ modulo $\mathcal{L}$ for a suitable subsequence $\left(k_{n}\right)_{n \in \mathbb{N}}$.

By Lemma 7.5, we have $y_{k_{n}} \subseteq r_{k_{n}}+\mathcal{\curlywedge}\left(-s_{k_{n}}+W\right)$. Hence, for all open neighbourhoods $U$ of 0 in $G$ and $V$ of 0 in $H$, there is some $N_{1}$ so that $\left(r_{k_{n}}, s_{k_{n}}\right)+\mathcal{L} \in\left(r^{\prime}+U, s^{\prime}+V\right)+\mathcal{L}$ holds for all $n>N_{1}$, and thus

$$
y_{k_{n}} \subseteq r^{\prime}+U+\curlywedge\left(-s^{\prime}-V+W\right) .
$$

Now, let $A>0$. Then, as $y_{k_{n}} \longrightarrow y$, there is some $N_{2}$ such that

$$
y \cap[-A, A]=y_{k_{n}} \cap[-A, A] \subseteq y_{k_{n}} \subseteq r^{\prime}+U+\curlywedge\left(-s^{\prime}-V+W\right)
$$

holds for all $n>N_{2}$. Since this applies to all $A>0$, we get $y \subseteq r^{\prime}+U+\curlywedge\left(-s^{\prime}-V+W\right)$.

Now, since we have this for all open neighbourhoods $U, V$ as specified, we have

$$
y \subseteq \bigcap_{U, V} r^{\prime}+U+\curlywedge\left(-s^{\prime}-V+W\right)=r^{\prime}+\curlywedge\left(-s^{\prime}+W\right)
$$

which shows that $-r^{\prime}+y \subseteq \curlywedge\left(-s^{\prime}+W\right)$. By the uniqueness of the parameter $(r, s)$ attached to $y \in \mathbb{Y}^{\prime}$, we get $\left(r^{\prime}, s^{\prime}\right)=(r, s)$ modulo $\mathcal{L}$ as desired.

At this point, for each character $\chi: \mathbb{A} \longrightarrow \mathbb{C}$, the mapping $\chi \circ \psi$ defines an eigenfunction of $(\mathbb{Y}, \mathbb{R}, \nu)$ that is continuous on $\mathbb{Y}^{\prime}$. This complements the statement of Proposition 7.4. We can now formulate the main result of this section as follows.

Theorem 7.8. The Kronecker factor of the dynamical system $(\mathbb{Y}, \mathbb{R}, \nu)$ can be identified with the MEF of the dynamical system obtained from the covering model set. It is explicitly given by $\mathbb{A}=(\mathbb{R} \times H) / \mathcal{L}$ within the $C P S(1)$, with $H=\mathbb{R}$.

Proof. The mapping $\psi: \mathbb{Y}^{\prime} \longrightarrow \mathbb{A}$ from above is the measure-theoretic factor map onto $\mathbb{A}$. The maximality of this factor is a consequence of Theorem 3.19, as the dual group of $\mathbb{A}$ is precisely the Fourier module of the pure point spectrum, which is tantamount to saying that the mappings $\chi \circ \psi$ on $\mathbb{Y}^{\prime}$ account for all eigenfunctions of our system.

Another approach, via a different view on the projection method, was recently suggested by Keller and Richard [27]. In this setting, as detailed in [28, Def. 2.2], the notion of an 'almost' MEF appears naturally, and is called a maximal equicontinuous generic factor, or MEGF for short. It is defined as

$$
\mathbb{Y}^{\prime \prime}:=\{y \in \mathbb{Y}: \overline{\mathbb{R}+y}=\mathbb{Y}\},
$$

which is closely related to the set $\mathbb{Y}^{\prime}$ defined earlier. Now, due to Corollary 7.6, we can apply $\left[28\right.$, Thm. 2.4 (iii)] to $\psi: \mathbb{Y}^{\prime} \cap \mathbb{Y}^{\prime \prime} \longrightarrow \mathbb{A}$ to derive that $\mathbb{A}$ is a factor of the MEGF, and 
that $\psi$ extends to a continuous mapping $\psi: \mathbb{Y}^{\prime \prime} \longrightarrow \mathbb{A}$. Since we already know that $\mathbb{A}$ is the Kronecker factor, being a factor of $\mathbb{Y}^{\prime \prime}$ means that it is metrically isomorphic to the MEGF.

In fact, one can see that $\mathbb{Y}^{\prime \prime} \subseteq \mathbb{Y}^{\prime}$ in this situation. If $y \in \mathbb{Y}^{\prime \prime}$ and $y_{0} \in Y_{0}$, we can find a sequence $\left(t_{n}\right)_{n \in \mathbb{N}}$ so that $t_{n}+y \longrightarrow y_{0}$ as $n \rightarrow \infty$. Since $y_{0}$ determines the window $W$ by Proposition 7.2, a computation similar to the ones at the end of Lemma 7.5 and Corollary 7.6 shows that $y \in \mathbb{Y}^{\prime}$. Putting the pieces together gives the following alternative view to our constructive approach.

Corollary 7.9. In the setting and notation of Theorem 7.8, the following assertions hold.

(1) $\mathbb{A}$ is the $M E G F$ of $\mathbb{Y}$, and $\psi: \mathbb{Y}^{\prime} \longrightarrow \mathbb{A}$ is continuous.

(2) The eigenfunctions of $(\mathbb{Y}, \mathbb{R}, \nu)$ are continuous on $\mathbb{Y}^{\prime}$.

(3) $(\mathbb{Y}, \mathbb{R}, \nu)$ is not weakly mixing.

7.3. Interpretation via disintegration. Now, consider the regular model set $\Lambda=\curlywedge(W)$ and the dynamical system obtained as the orbit closure under the $\mathbb{R}$-action. This is a uniquely ergodic system with pure point spectrum, and it is a.e. 1: 1 over its MEF, which is a 2-torus in our case at hand. This one also acts as the Kronecker factor for our system $(\mathbb{Y}, \mathbb{R}, \nu)$, where the map is only defined for $\nu$-almost every element of $\mathbb{Y}$ by first identifying the unique covering model set and then projecting down to the MEF.

The MEF of the covering model set is the compact Abelian group $\mathbb{A}$, which is the 2-torus equipped with Lebesgue measure as its Haar measure. Here, the translation action is represented by a group addition with dense range, as mentioned earlier. Now, over every $a \in \mathbb{A}$, we have a fibre $Y_{a} \subset \mathbb{Y}$ together with a probability measure $\nu_{a}$ on it. For $a=0$, this is just our special fibre $Y_{0}$ from above. These fibre measures are compatible with the (normalised) Haar measure on $\mathbb{A}$ as needed for a disintegration formula. For our dynamical system $(\mathbb{Y}, \mathbb{R}, \nu)$ and any $f \in L^{1}(\mathbb{Y}, \nu)$, we then have

$$
\mathbb{E}(f)=\int_{\mathbb{Y}} f(y) \mathrm{d} \nu(y)=\int_{\mathbb{A}} \int_{Y_{a}} f(y) \mathrm{d} \nu_{a}(y) \mathrm{d} a=\int_{\mathbb{A}} \mathbb{E}\left(f \mid Y_{a}\right) \mathrm{d} a,
$$

in line with the general theory; see [21, Ch. 5.4].

Analogous expressions hold for measure-valued quantities. When the inner integral is translation invariant on $\mathbb{A}$, as is the case for the autocorrelation and diffraction measures encountered earlier, the expectation can be obtained from the conditional expectation over one fibre, say $Y_{0}$, which is precisely the approach taken in the previous sections.

Remark 7.10. The analogous procedure also works, step by step, for the random noble means inflations from Section 4. The topological point spectrum is once again trivial. Here, the proof uses the existence of elements $y_{1}$ and $y_{2}$ in the special fibre $Y_{0}$ such that $1+y_{1}$ as well as $\lambda_{m}+y_{2}$ also lie in $Y_{0}$, with the same conclusion as before; see [42] for details. Also, non-trivial eigenfunctions are only discontinuous on a null set.

Moreover, the entire structure with the covering model set and its dynamical system carries over, thus establishing the MEF of the model set as the Kronecker factor of $(\mathbb{Y}, \mathbb{R}, \nu)$, and as its MEGF. The disintegration then works the same way as in the Fibonacci case. 
7.4. Random period doubling chain. Here, the situation is slightly different for two reasons. First, the substitution (26) is of constant length, which means that we can identify the discrete and the tiling picture and work with the $\mathbb{Z}$-action of the shift. Second, the connection to a model set requires a 2-adic internal space, so that handling windows is more complicated.

Let $\mathbb{X}_{0}$ denote the discrete hull, and $\left(\mathbb{X}_{0}, \mathbb{Z}, \nu\right)$ the corresponding dynamical system, with $\nu$ denoting the patch frequency measure, which is ergodic. As before, $\mathbb{X}_{0}$ contains a special fibre, denoted $X_{0}$, which contains all elements that are realisations in the form of two level- $\infty$ supertiles (or superwords) meeting at 0 . Here, the eigenfunction equation takes the form

$$
f(n+x)=\mathrm{e}^{2 \pi \mathrm{i} k n} f(x)
$$

for some $k \in \mathbb{T}$, the dual group of $\mathbb{Z}$, and then all $n \in \mathbb{Z}$. We represent $\mathbb{T}$ as the half-open interval $[0,1)$ with addition modulo 1 .

Proposition 7.11. The topological point spectrum of $\left(\mathbb{X}_{0}, \mathbb{Z}, \nu\right)$ is trivial, which is to say that the only continuous eigenfunction is the constant one.

Proof. Let $f$ be a continuous eigenfunction. As in the proof of Proposition 7.4, $|f|$ is continuous and invariant and hence constant (as $\left(\mathbb{X}_{0}, \mathbb{Z}, \nu\right)$ is ergodic). Moreover, $f$ is again constant on the special fibre $X_{0}$.

Next, observe that $\mathbb{X}_{0}$ contains a periodic element, namely the one obtained by periodic repetition of the 3 -letter word $a a b$. What is more, it is contained in the fibre $X_{0}$ in three different ways, as is apparent from

$$
\begin{aligned}
& \cdots \underline{b a} \underline{a b} \underline{a a} \underline{b a} \underline{a b} \mid \underline{a a} \underline{b a} \underline{a b} \underline{a b} \underline{a a} \cdots \\
& \cdots \underline{a b} \underline{a a} \underline{b a} \underline{a b} \underline{a a} \mid \underline{b a} \underline{a b} \underline{a a} \underline{b a} \underline{a b} \cdots \\
& \cdots \underline{a a} \underline{b a} \underline{a b} \underline{a a} \underline{b a} \mid \underline{a b} \underline{a a} \underline{b a} \underline{a b} \underline{a a} \cdots .
\end{aligned}
$$

Since $f$ takes the same value on all three, which are translates of one another, we get

$$
\mathrm{e}^{2 \pi \mathrm{i} k}=1 \quad \text { with } k \in \mathbb{T}
$$

which implies $k=0$. Thus, $f$ must be the constant eigenfunction as claimed.

As we can already see from our diffraction analysis in Section 6, the measure-theoretic point spectrum of $\left(\mathbb{X}_{0}, \mathbb{Z}, \nu\right)$ is given by $\mathbb{T} \cap \mathbb{Z}\left[\frac{1}{2}\right]$. As before, $\mathbb{X}_{0}$ contains an open set of full measure, $\mathbb{X}_{0}^{\prime}$ say, with the property that all eigenfunctions are continuous on it. The discontinuity is thus once again caused by a null set in the hull.

Remark 7.12. Via a suspension with a constant roof function, the discrete dynamical system $\left(\mathbb{X}_{0}, \mathbb{Z}, \nu\right)$ can be embedded into a flow, written as $\left(\mathbb{X}, \mathbb{R}, \nu_{\mathbb{R}}\right)$ with $\nu_{\mathbb{R}}$ being the standard extension of $\nu$ to an invariant probability measure on $\mathbb{X}$. This system is also ergodic, and the topological point spectrum becomes $\mathbb{Z}$, while the measure-theoretic point spectrum is all of $\mathbb{Z}\left[\frac{1}{2}\right]$. The additional continuous eigenfunctions in comparison to Proposition 7.11 trivially emerge from the suspension. In terms of the approach via the Fourier-Bohr coefficients, this 
can be seen by adding a complex weight of the form $\mathrm{e}^{2 \pi \mathrm{ink}}$ with a fixed $n \in \mathbb{Z}$, which results in a phase change for the continuous flow, but remains invisible for the discrete shift.

From here, the remainder of the argument is similar to before. We get a covering twocomponent model set, and its $\mathrm{MEF}$ as the Kronecker factor of $\left(\mathbb{X}, \mathbb{R}, \nu_{\mathbb{R}}\right)$. Moreover, there exists a continuous mapping from the set $\mathbb{X}^{\prime}$ to the Kronecker factor, and $\mathbb{X}^{\prime}$ contains all elements with dense orbit. In particular, the Kronecker factor is also the MEGF, and all eigenfunctions are continuous on $\mathbb{X}^{\prime}$. As a consequence, we also have the disintegration as in Eq. (36), which explains the nice formulas we were able to obtain in Section 6. At this point, we leave further details to the interested reader.

\section{OUtLOOK}

The focus of this article was on compatible substitutions or inflations that are ultimately related to a regular model set via an implicit thinning process. This made the spectral structure fully accessible. In general, the situation will be more complex, in particular as far as the relation between the topological point spectrum and the measure-theoretic one is concerned. Thus, an approach in several steps seems most promising.

First, one could consider semi-compatible inflations that still share the same substitution matrix, but do no longer define the same hull; for one concrete example, where the complete determination of the diffraction measure is still possible, we refer to [23]. Next, one could relax the connection to model sets, and consider local mixtures of substitutions with singular continuous spectrum. As long as they still share the left PF eigenvector of the substitution matrix, concrete results should still be possible because a consistent geometric realisation exists under this condition.

Whether more general mixtures (such as one between the Fibonacci and the Thue-Morse

substitution, which is sometimes called 'Fib-Morse') will lead to reasonable results is presently unclear, but somewhat doubtful. Prior to such an attempt, a better understanding of the general structure of random substitutions and their hulls is needed, where we refer to [40] for some first systematic steps.

\section{APPENDiX}

In several places in the main manuscript, we have used approximation results for the autocorrelation of a translation bounded pure point measure. Our concrete justification for these steps was based on a certain type of uniform convergence of the approximating measures on finite regions of growing size (in fact, we have used a one-sided version of such an approximation). Clearly, the concrete criteria were sufficient, but certainly not necessary. In this appendix, we look at this situation in a slightly more systematic way. We do this first for an approximation by unbounded (but still translation bounded) measures, and then for the case that one uses finite measures as approximations, which is the typical scenario in inflation-based systems. 
Below, we formulate our results for measures in $\mathbb{R}^{d}$, and refer to [12] for a more general setting. Note that there is also a complementary selection of results on the Fourier side of the coin, that is, there are several approximation results for the diffraction measure of a given translation bounded measure $\omega \in \mathcal{M}^{\infty}\left(\mathbb{R}^{d}\right)$. Though this is both interesting and relevant in its own right, we concentrate on the autocorrelation measures here, and refer to $[4,12]$ for results on their Fourier transforms.

8.1. Approximation by unbounded measures. Consider a sequence $\left(\mu_{n}\right)_{n \in \mathbb{N}}$ of translation bounded measure such that $\mu_{n} \stackrel{n \rightarrow \infty}{\longrightarrow} \mu$ in the vague topology. Assume that a van Hove sequence $\mathcal{A}=\left(A_{m}\right)_{m \in \mathbb{N}}$ is given such that the autocorrelations $\gamma_{n}=\mu_{n} \circledast \widetilde{\mu_{n}}$ and $\gamma=\mu \circledast \widetilde{\mu}$ all exist along $\mathcal{A}$. When is it true that also $\gamma=\lim _{n \rightarrow \infty} \gamma_{n}$ holds? That this must fail in general can easily be seen from an example, such as $\mu_{n}=\delta_{2 \mathbb{Z}}+\delta_{(2 \mathbb{Z}+1) \cap[-n, n]}$. Here, one has $\gamma=\mu=\delta_{\mathbb{Z}}$, but $\gamma_{n}=\frac{1}{2} \delta_{2 \mathbb{Z}}$, for all $n \in \mathbb{N}$. It is thus clear that one needs some other relation between $\mu$ and $\mu_{n}$. In particular, as we shall see, we do not need vague convergence, while convergence in a different topology is what counts. This is common in the diffraction context, as nicely outlined in [34].

Let a van Hove sequence $\mathcal{A}$ be fixed, with all $A_{m}$ compact. We assume $A_{m} \subset A_{m+1}$, together with the usual condition that $\operatorname{vol}\left(\partial^{K} A_{m}\right)=\mathcal{O}\left(\operatorname{vol}\left(A_{m}\right)\right)$ as $m \rightarrow \infty$ for any compact $K \subset \mathbb{R}^{d}$, where $\partial^{K} S$ is the $K$-boundary of the set $S$; see [4] for details. There are various possible generalisations of this setting, which we omit here. We need $\mathcal{A}$ for averages of various kinds, such as the volume-averaged or Eberlein convolution $f \circledast g$ of two (locally integrable) functions $f$ and $g$, as (pointwise) given by

$$
(f \circledast g)(x):=\lim _{m \rightarrow \infty} \frac{\left(\left.\left.f\right|_{A_{m}} * g\right|_{A_{m}}\right)(x)}{\operatorname{vol}\left(A_{m}\right)},
$$

whenever this limit exists. Here, $\left.f\right|_{A_{m}}$ denotes the restriction of $f$ to $A_{m}$ and $*$ the ordinary convolution of functions. Operations of this type are needed when dealing with almost periodic functions and related objects. Note that $f \circledast g=0$ if $f$ or $g$ has compact support.

Let $C_{\mathrm{u}}\left(\mathbb{R}^{d}\right)$ denote the space of uniformly continuous and bounded functions. The mean of $f \in C_{\mathbf{u}}\left(\mathbb{R}^{d}\right)$ relative to $\mathcal{A}$ is defined as

$$
M(f):=\lim _{m \rightarrow \infty} \frac{1}{\operatorname{vol}\left(A_{m}\right)} \int_{A_{m}} f(x) \mathrm{d} x,
$$

provided the limit exists. This is certainly the case for all weakly almost periodic functions, but not for all $f \in C_{\mathbf{u}}\left(\mathbb{R}^{d}\right)$. In contrast, one can define the upper absolute mean along $\mathcal{A}$ as

$$
\bar{M}(f):=\limsup _{m \rightarrow \infty} \frac{1}{\operatorname{vol}\left(A_{m}\right)} \int_{A_{m}}|f(x)| \mathrm{d} x,
$$

which clearly exists for all $f \in C_{\mathrm{u}}\left(\mathbb{R}^{d}\right)$ and satisfies $\bar{M}(f)=\bar{M}(|f|)$. This way, $\bar{M}($.$) defines$ a semi-norm on $C_{\mathrm{u}}\left(\mathbb{R}^{d}\right)$. There are obvious variants of this definition, but we only need this simple version below. Here, a sequence $\left(f_{n}\right)_{n \in \mathbb{N}}$ of functions from $C_{\mathrm{u}}\left(\mathbb{R}^{d}\right)$ is mean convergent to $g \in C_{\mathrm{u}}\left(\mathbb{R}^{d}\right)$ if $\bar{M}\left(f_{n}-g\right) \stackrel{n \rightarrow \infty}{\longrightarrow} 0$. We use $f_{n} \rightsquigarrow g$ to denote this type of convergence. 
Now, let $f, g, h \in C_{\mathbf{u}}\left(\mathbb{R}^{d}\right)$ and assume that both $f \circledast h$ and $g \circledast h$ exist relative to $\mathcal{A}$, as defined by Eq. (37). It is elementary to verify the estimate

$$
\|f \circledast h-g \circledast h\|_{\infty} \leqslant \bar{M}(f-g)\|h\|_{\infty},
$$

which has the following important consequence.

Fact 8.1. Let $f_{n}, g, h \in C_{\mathrm{u}}\left(\mathbb{R}^{d}\right)$, where $n \in \mathbb{N}$. Assume that $f_{n} \rightsquigarrow g$ as $n \rightarrow \infty$ and that $g \circledast h$ as well as $f_{n} \circledast h$ exists for all $n \in \mathbb{N}$ with respect to a given van Hove sequence $\mathcal{A}$. Then, one has $\lim _{n \rightarrow \infty}\left\|f_{n} \circledast h-g \circledast h\right\|_{\infty}=0$.

The crucial observation here is that the convergence in mean for the $f_{n}$ implies a much stronger type of convergence after Eberlein convolution. It is a known phenomenon that the Eberlein convolution usually has nicer properties than the original functions. Indeed, the Eberlein convolution of two functions is weakly almost periodic, and the Eberlein convolution of two weakly almost periodic functions becomes uniformly (or Bohr) almost periodic.

Let $\mathcal{M}^{\infty}\left(\mathbb{R}^{d}\right)$ denote the space of translation bounded Radon measures on $\mathbb{R}^{d}$, which we primarily see as continuous linear functionals over $C_{\mathrm{c}}\left(\mathbb{R}^{d}\right)$, the space of continuous functions with compact support, but we also identify Radon measures with regular measures over the Borel $\sigma$-algebra by the general Riesz-Markov representation theorem for this case [18]. Now, given a sequence $\left(\mu_{n}\right)_{n \in \mathbb{N}}$ of measures from $\mathcal{M}^{\infty}\left(\mathbb{R}^{d}\right)$, we need convergence in different topologies. The standard one is vague convergence, denoted by $\mu_{n} \rightarrow \mu$, which means $\lim _{n \rightarrow \infty} \mu_{n}(f)=\mu(f)$ for all $f \in C_{\mathrm{c}}\left(\mathbb{R}^{d}\right)$. Since $\mu(f)=\left(\mu * f_{-}\right)(0)$, where $f_{-}(x)=f(-x)$, one can equivalently characterise vague convergence via $\lim _{n \rightarrow \infty}\left(\mu_{n} * g\right)(0)=(\mu * g)(0)$ for all $g \in C_{\mathrm{c}}\left(\mathbb{R}^{d}\right)$. It is easy to see that this is actually equivalent to the seemingly stronger $\mu_{n} * g \stackrel{n \rightarrow \infty}{\longrightarrow} \mu * g$ pointwise on $\mathbb{R}^{d}$, for all $g \in C_{\mathrm{c}}\left(\mathbb{R}^{d}\right)$.

Next, we speak of norm convergence to a measure $\mu \in \mathcal{M}^{\infty}\left(\mathbb{R}^{d}\right)$, denoted by $\mu_{n} \Rightarrow \mu$, if $\left\|\mu_{n}-\mu\right\|_{K} \stackrel{n \rightarrow \infty}{\longrightarrow} 0$ for some (fixed) compact set $\varnothing \neq K \subset \mathbb{R}^{d}$ that is the closure of its interior, where $\|\mu\|_{K}:=\sup _{t \in \mathbb{R}^{d}}|\mu|(t+K)$. Note that any $K$ with $\varnothing \neq K=\overline{K^{\circ}}$ defines the same topology. Next, we speak of convergence in the product topology, denoted by $\mu_{n} \stackrel{\pi}{\rightarrow} \mu$, if one has $\left\|\left(\mu_{n}-\mu\right) * g\right\|_{\infty} \stackrel{n \rightarrow \infty}{\longrightarrow} 0$ for all $g \in C_{\mathrm{c}}\left(\mathbb{R}^{d}\right)$; and of mean convergence, as before denoted by $\mu_{n} \rightsquigarrow \mu$, if $\mu_{n} * g \rightsquigarrow \mu * g$ as $n \rightarrow \infty$ holds for all $g \in C_{\mathrm{c}}\left(\mathbb{R}^{d}\right)$. The topology induced by mean convergence can also be induced by the family of semi-norms given by $|\mu|_{g}:=\bar{M}(\mu * g)$ with $g \in C_{\mathrm{c}}\left(\mathbb{R}^{d}\right)$. It is not a Hausdorff topology.

Via standard estimates, one can now verify the following relations.

Lemma 8.2. For translation bounded measures $\mu_{n}$, with $n \in \mathbb{N}$, and $\mu$, one has the implications

$$
\left(\mu_{n} \Rightarrow \mu\right) \Longrightarrow\left(\mu_{n} \stackrel{\pi}{\rightarrow} \mu\right) \Longrightarrow\left\{\begin{array}{l}
\left(\mu_{n} \rightsquigarrow \mu\right) \\
\left(\mu_{n} \rightarrow \mu\right)
\end{array}\right.
$$

none of which is reversible in general. Moreover, considering mean versus vague convergence, one has that neither of them implies the other. 
Let us give a few examples to illustrate Lemma 8.2. As $n \rightarrow \infty$, one has $\delta_{1 / n} \stackrel{\pi}{\rightarrow} \delta_{0}$, but no convergence in the norm topology. Likewise, $\delta_{n} \rightarrow 0$ and $\delta_{n} \rightsquigarrow 0$, but no convergence in the product topology. The measures $\mu_{n}=\delta_{2 \mathbb{Z}}+\delta_{(2 \mathbb{Z}+1) \cap[-n, n]}$ from above satisfy $\mu_{n} \rightarrow \delta_{\mathbb{Z}}$ and $\mu_{n} \rightsquigarrow \delta_{2 \mathbb{Z}}$. Note that the last relation could change if we replace Eq. (38) by some of the possible variants. In this sense, one has to be careful with the possible notions of mean convergence [12].

Remark 8.3. There are important further relations between the above topologies if one restricts them to suitable subclasses of translation bounded measures. Let us simply mention some of them, and refer to [12] for proofs and further details, in the more general setting of locally compact Abelian groups. Here, let $\Lambda \subset \mathbb{R}^{d}$ be a uniformly discrete point set, and select an open neighbourhood $U \subset \mathbb{R}^{d}$ of 0 such that, for any $x, y \in \Lambda$ with $x \neq y$, the sets $x+U$ and $y+U$ are disjoint. Further, fix a compact set $K \subset U$ with non-empty interior that also satisfies $K=-K$, which is clearly possible.

Now, consider pure point measures $\mu, \mu_{n} \in \mathcal{M}^{\infty}\left(\mathbb{R}^{d}\right)$, with $n \in \mathbb{N}$, which are all supported in $\Lambda$. For any $f \in C_{\mathrm{c}}\left(\mathbb{R}^{d}\right)$ with $\operatorname{supp}(f) \subset U$, we then have $\left|\left(\mu_{n}-\mu\right) * f\right|=\left|\mu_{n}-\mu\right| *|f|$ by [43, Lemma 5.8.3]. If $f$ is chosen such that $1_{K} \leqslant f \leqslant 1_{U}$, one can show by standard estimates that $\left\|\mu_{n}-\mu\right\|_{K} \leqslant\left\|\left(\mu_{n}-\mu\right) * f\right\|_{\infty}$. Together with Lemma 8.2, this implies the equivalence of norm convergence and convergence in the product topology for such measures, which can be extremely useful in the diffraction context, because the spectral type is preserved under norm convergence [4, Thm. 8.4].

Next, one has $\left\|\mu_{n}-\mu\right\|_{K}=\sup _{x \in \Lambda}\left|\mu_{n}-\mu\right|(\{x\})$ under the same assumptions on the measures $\mu$ and $\mu_{n}$, and norm convergence, and hence product convergence, is equivalent to uniform convergence $\mu_{n}(\{x\}) \stackrel{n \rightarrow \infty}{\longrightarrow} \mu(\{x\})$ for $x \in \Lambda$. In contrast, in this setting, simple pointwise convergence for $x \in \Lambda$ is only equivalent to vague convergence, $\mu_{n} \rightarrow \mu$.

Finally, for $f \in C_{\mathrm{c}}\left(\mathbb{R}^{d}\right)$, one has $\bar{M}\left(\left(\mu_{n}-\mu\right) * f\right) \leqslant\|f\|_{1} \bar{M}\left(\mu_{n}-\mu\right)$, where

$$
\bar{M}(\mu):=\limsup _{n \rightarrow \infty} \frac{|\mu|\left(A_{n}\right)}{\operatorname{vol}\left(A_{n}\right)} .
$$

If $\operatorname{supp}(f) \subset U$, one gets equality in the previous estimate, which shows that $\mu_{n} \rightsquigarrow \mu$ is equivalent with $\bar{M}\left(\mu_{n}-\mu\right) \rightarrow 0$.

Behind all these relations, maybe somewhat implicitly, is the observation that the structure of the autocorrelation imposes a natural topology on the dynamical system itself, as explained in more detail in $[35,34,11]$. The key result of this section can now be stated as follows.

Theorem 8.4. Let $\mu_{n}$ with $n \in \mathbb{N}$ and $\mu$ be equi-translation bounded measures, and assume that their autocorrelations $\gamma_{n}=\mu_{n} \circledast \widetilde{\mu_{n}}$ and $\gamma=\mu \circledast \widetilde{\mu}$ exist for all $n \in \mathbb{N}$. Assume further that also the Eberlein convolutions $\mu_{n} \circledast \mu$ exist.

Then, if $\mu_{n} \rightsquigarrow \mu$, one also has $\gamma_{n} \stackrel{\pi}{\rightarrow} \gamma$. In particular, one then has $\lim _{n \rightarrow \infty} \gamma_{n}=\gamma$ in the vague topology. 
Proof. Let $f, g \in C_{\mathrm{c}}\left(\mathbb{R}^{d}\right)$ be arbitrary, but fixed. Due to the assumed equi-translation boundedness, there is a constant $c$, which may depend on $f$ and $g$, so that $\|\mu * f\|_{\infty} \leqslant c$ together with $\left\|\mu_{n} * g\right\|_{\infty} \leqslant c$ for all $n \in \mathbb{N}$.

Now, we can estimate as follows,

$$
\begin{aligned}
\left\|\gamma * f * \widetilde{g}-\gamma_{n} * f * \widetilde{g}\right\|_{\infty}= & \left\|(\mu * f) \circledast \widetilde{(\mu * g)}-\left(\mu_{n} * f\right) \circledast\left(\widetilde{\mu_{n} * g}\right)\right\|_{\infty} \\
\leqslant & \|(\mu * f) \circledast \widetilde{(\mu * g)}-(\mu * f) \circledast\left(\widetilde{\left.\mu_{n} * g\right)} \|_{\infty}\right. \\
& +\left\|(\mu * f) \circledast \widetilde{\left(\mu_{n} * g\right)}-\left(\mu_{n} * f\right) \circledast\left(\widetilde{\mu_{n} * g}\right)\right\|_{\infty} \\
\leqslant & \bar{M}\left(\widetilde{\mu * g}-\widetilde{\mu_{n} * g}\right)\|\mu * f\|_{\infty}+\bar{M}\left(\mu * f-\mu_{n} * f\right)\left\|\mu_{n} * g\right\|_{\infty} \\
\leqslant & c\left(\bar{M}\left(\left(\mu-\mu_{n}\right) * g\right)+\bar{M}\left(\left(\mu-\mu_{n}\right) * f\right)\right),
\end{aligned}
$$

where Eq. (39) was used in the penultimate step.

This way, we get $\gamma_{n} \stackrel{\pi}{\rightarrow} \gamma$, and hence $\gamma_{n} \rightarrow \gamma$, from $\mu_{n} \rightsquigarrow \mu$ in conjunction with the wellknown fact that linear combinations of functions of the form $f * \widetilde{g}$, with $f, g \in C_{\mathrm{c}}\left(\mathbb{R}^{d}\right)$, are dense in $C_{\mathrm{c}}\left(\mathbb{R}^{d}\right)$.

In particular, we see that we do not need vague convergence of $\mu_{n}$ to $\mu$, but rather convergence in the mean, which is both weaker and stronger in some sense.

8.2. Approximation by bounded measures. Now, let us consider a sequence $\left(\mu_{n}\right)_{n \in \mathbb{N}}$ of finite measures such that $\lim _{n \rightarrow \infty} \mu_{n}=\omega$ holds in the vague topology, where $\omega$ is some fixed translation bounded measure, with autocorrelation $\gamma_{\omega}=\omega \circledast \widetilde{\omega}$ relative to a given van Hove averaging sequence $\mathcal{A}$ as before. Let us assume that the sets $A_{n}$ are chosen such that they can also serve as supporting sets for the $\mu_{n}$. Now, our previous question can be rephrased as follows: When is it true that

$$
\gamma_{\omega}=\lim _{n \rightarrow \infty} \frac{\mu_{n} * \widetilde{\mu_{n}}}{\operatorname{vol}\left(A_{n}\right)}
$$

holds? The difference to before is that we cannot define autocorrelations for the finite measures $\mu_{n}$ along $\mathcal{A}$. However, under our assumptions, we may compare $\mu_{n} * \widetilde{\mu_{n}}$ with $\omega_{n} * \widetilde{\omega_{n}}$, where $\omega_{n}:=\left.\omega\right|_{A_{n}}$ is the restriction of $\omega$ to the set $A_{n}$. Note that, in general, $\mu_{n} \neq \omega_{n}$, and our task is to control the difference

$$
\frac{1}{\operatorname{vol}\left(A_{n}\right)}\left(\mu_{n} * \widetilde{\mu_{n}}-\omega_{n} * \widetilde{\omega_{n}}\right)=\frac{1}{\operatorname{vol}\left(A_{n}\right)}\left(\mu_{n} *\left(\widetilde{\mu_{n}}-\widetilde{\omega_{n}}\right)+\left(\mu_{n}-\omega_{n}\right) * \widetilde{\omega_{n}}\right) .
$$

Here, we need a modified concept of mean convergence. Assume for simplicity that the $A_{m}$ are nice compact sets, say convex, such that $\mu_{n}$ agrees with its restriction to $A_{m}$ for all $m \geqslant n$, (but not for smaller $m$ ). Then, with $\nu_{n}=\mu_{n}-\omega_{n}$, we can say that $\nu_{n}$, with $\operatorname{supp}\left(\nu_{n}\right) \subseteq A_{n}$, converges in mean to 0 , denoted by $\nu_{n} \rightsquigarrow 0$ in analogy to above, if

$$
\limsup _{n \rightarrow \infty} \frac{1}{\operatorname{vol}\left(A_{n}\right)} \int_{K+A_{n}}\left|\nu_{n} * g\right|(x) \mathrm{d} x=0
$$


holds for every $g \in C_{\mathrm{c}}\left(\mathbb{R}^{d}\right)$, with $K=K_{g}$ denoting the compact support of $g$. Note that this definition makes sense because, for any given $g$ (and hence $K$ ), the van Hove property of $\mathcal{A}$ implies that $\operatorname{vol}\left(K+A_{n}\right)=\mathcal{O}\left(\operatorname{vol}\left(A_{n}\right)\right)$ for large $n$. Also, one could equally well use $\lim _{n \rightarrow \infty}$ instead of $\limsup _{n \rightarrow \infty}$ in Eq. (40).

Remark 8.5. Via a standard estimate in conjunction with a Fubini-type argument, one can see that, for any $g \in C_{\mathrm{c}}\left(\mathbb{R}^{d}\right)$, Eq. (40) follows from

$$
\lim _{n \rightarrow \infty} \frac{\left|\nu_{n}\right|\left(A_{n}\right)}{\operatorname{vol}\left(A_{n}\right)}=0
$$

which looks perhaps like a more natural way to define mean convergence to 0 . However, this is a generally stronger notion, wherefore we prefer to use the above version.

When the measures $\nu_{n}$ are equi-translation bounded, one can show that the condition in (40) is equivalent to

$$
\lim _{n \rightarrow \infty} \frac{1}{\operatorname{vol}\left(A_{n}\right)} \int_{A_{n}}\left|\nu_{n} * g\right|(x) \mathrm{d} x=0,
$$

which can be proved on the basis of the van Hove property of $\mathcal{A}$. If, in addition, all $\nu_{n}$ are supported in a uniformly discrete set $\Lambda \subset \mathbb{R}^{d}$, the conditions from Eq. (40) become equivalent to Eq. (41); for details, see [12].

The crucial point for the approach with finite measures is the mutual adjustment of the supports of the approximating measures with the elements of the van Hove sequence $\mathcal{A}$. In practice, this is usually done by selecting $\mathcal{A}$ according to the approximating measures, which often originate naturally, for instance from an inflation rule or a similar process.

The main result of this section now reads as follows.

Theorem 8.6. Let $\omega \in \mathcal{M}^{\infty}\left(\mathbb{R}^{d}\right)$, and assume that its autocorrelation, $\gamma_{\omega}$, exists for a given van Hove sequence $\mathcal{A}$, so

$$
\gamma_{\omega}=\omega \circledast \widetilde{\omega}=\lim _{n \rightarrow \infty} \frac{\omega_{n} * \widetilde{\omega_{n}}}{\operatorname{vol}\left(A_{n}\right)}
$$

with $\omega_{n}:=\left.\omega\right|_{A_{n}}$. Let $\left(\mu_{n}\right)_{n \in \mathbb{N}}$ be a sequence of finite measures with $\operatorname{supp}\left(\mu_{n}\right) \subseteq A_{n}$, and assume that the $\mu_{n}$ are equi-translation bounded and satisfy $\left(\mu_{n}-\omega_{n}\right) \rightsquigarrow 0$ as $n \rightarrow \infty$.

Then, $\frac{1}{\operatorname{vol}\left(A_{n}\right)}\left(\mu_{n} * \widetilde{\mu_{n}}-\omega_{n} * \widetilde{\omega_{n}}\right) \stackrel{\pi}{\rightarrow} 0$ as $n \rightarrow \infty$. In particular, one has

$$
\lim _{n \rightarrow \infty} \frac{\mu_{n} * \widetilde{\mu_{n}}}{\operatorname{vol}\left(A_{n}\right)}=\gamma_{\omega}
$$

in the vague topology.

Proof. Since $\omega$ is translation bounded, the finite measures $\omega_{n}$ are equi-translation bounded. Now, given $f, g \in C_{\mathrm{c}}\left(\mathbb{R}^{d}\right)$, the assumed equi-translation boundedness of the measures $\mu_{n}$ implies the existence of a constant $c$, which may depend on $f$ and $g$, such that $\left\|\mu_{n} * f\right\|_{\infty} \leqslant c$ and $\left\|\omega_{n} * g\right\|_{\infty} \leqslant c$ for all $n \in \mathbb{N}$. 
Now, with $\|\widetilde{h}\|_{\infty}=\|h\|_{\infty}$ for any $h \in C_{\mathrm{c}}\left(\mathbb{R}^{d}\right)$, we get

$$
\begin{aligned}
& \left\|\mu_{n} * \widetilde{\mu_{n}} * f * \widetilde{g}-\omega_{n} * \widetilde{\omega_{n}} * f * \widetilde{g}\right\|_{\infty} \\
& \quad \leqslant\left\|\left(\mu_{n} * f\right) *\left(\widetilde{\mu_{n}}-\widetilde{\omega_{n}}\right) * \widetilde{g}\right\|_{\infty}+\left\|\left(\mu_{n}-\omega_{n}\right) * f *\left(\widetilde{\omega_{n} * g}\right)\right\|_{\infty} \\
& \quad \leqslant\left\|\mu_{n} * f\right\|_{\infty} \int_{\mathbb{R}^{d}}\left|\left(\widetilde{\mu_{n}}-\widetilde{\omega_{n}}\right) * \widetilde{g}\right|(x) \mathrm{d} x+\left\|\omega_{n} * g\right\|_{\infty} \int_{\mathbb{R}^{d}}\left|\left(\mu_{n}-\omega_{n}\right) * f\right|(x) \mathrm{d} x \\
& \quad \leqslant c \int_{K+A_{n}}\left|\left(\mu_{n}-\omega_{n}\right) * f\right|(x)+\left|\left(\mu_{n}-\omega_{n}\right) * g\right|(x) \mathrm{d} x,
\end{aligned}
$$

where the compact set $K$ is chosen such that it contains the supports of $f$ and $g$. Dividing both sides by $\operatorname{vol}\left(A_{n}\right)$, the claim follows from the assumption of mean convergence and Eq. (40), in complete analogy to our previous result.

The main difference to the arguments used in the main text is the replacement of a uniform condition by a condition in mean, which should be useful under more general circumstances.

\section{ACKNOWLEDGEMENTS}

It is a pleasure to thank Philipp Gohlke, Uwe Grimm, Gerhard Keller and Dan Rust for helpful discussions. We thank an anonymous reviewer for a number of suggestions that helped to improve the manuscript. This work was supported by the German Research Council (DFG), within the CRC 1283, and by Natural Sciences and Engineering Council of Canada (NSERC), via grant 03762-2014. Moreover, a research stay of N.S. was partially supported by the Simons Foundation and by the Oberwolfach Research Institute for Mathematics (MFO).

\section{REFERENCES}

[1] L. Argabright and J. Gil de Lamadrid, Fourier analysis of unbounded measures on locally compact Abelian groups, Memoirs AMS no. 145, AMS, Providence, RI (1974).

[2] M. Baake, M. Birkner and R.V. Moody, Diffraction of stochastic point sets: Explicitly computable examples, Commun. Math. Phys. 293 (2010), 611-660; arXiv:0803.1266.

[3] M. Baake, F. Gähler and U. Grimm, Spectral and topological properties of a family of generalised Thue-Morse sequences, J. Math. Phys. 53 (2012), 032701, 1-24; arXiv:1201.1423.

[4] M. Baake and U. Grimm, Aperiodic Order. Vol. 1: A Mathematical Invitation, Cambridge University Press, Cambridge (2013).

[5] M. Baake, H. Kösters and R.V. Moody, Diffraction theory of point processes: Systems with clumping and repulsion, J. Stat. Phys. 159 (2015), 915-936; arXiv:1405.4255.

[6] M. Baake and D. Lenz, Dynamical systems on translation bounded measures: Pure point dynamical and diffraction spectra, Ergod. Th. E Dynam. Syst. 24 (2005), 1867-1893; arXiv:math.DS/0302061.

[7] M. Baake and D. Lenz, Deformation of Delone dynamical systems and pure point diffraction, J. Fourier Anal. Appl. 11 (2005), 125-150; arXiv:math.DS/0404155.

[8] M. Baake, D. Lenz and R.V. Moody, Characterization of model sets by dynamical systems, Ergodic Th. ES Dynam. Syst. 27 (2007), 341-382; arXiv:math.DS/0511648.

[9] M. Baake, D. Lenz and C. Richard, Pure point diffraction implies zero entropy for Delone sets with uniform cluster frequencies, Lett. Math. Phys. 82 (2007), 61-77; arXiv:0706.1677. 
[10] M. Baake and R.V. Moody, Self-similar measures for quasicrystals, in Directions in Mathematical Quasicrystals, eds. M. Baake and R.V. Moody, CRM Monograph Series, vol. 13, AMS, Providence, RI (2000), pp. 1-42.

[11] M. Baake and R.V. Moody, Weighted Dirac combs with pure point diffraction, J. reine angew. Math. (Crelle) $\mathbf{5 7 3}$ (2004), 61-94; arXiv:math.MG/0203030.

[12] M. Baake, T. Spindeler and N. Strungaru, Approximation and decomposition of autocorrelation measures, in preparation.

[13] M.F. Barnsley, Lecture notes on iterated function systems, in Chaos and Fractals, eds. R.L. Devaney and L. Keen, Proc. Symp. Appl. Math. vol. 39, AMS, Providence, RI (1989), pp. 127-144.

[14] G. Bernuau and M. Duneau, Fourier analysis of deformed model sets, in Directions in Mathematical Quasicrystals, eds. M. Baake and R.V. Moody, CRM Monograph Series, vol. 13, AMS, Providence, RI (2000), pp. 43-60.

[15] C. Berg and G. Forst, Potential Theory on Locally Compact Abelian Groups, Springer, Berlin (1975).

[16] M. Brin and G. Stuck, Introduction to Dynamical Systems, Cambridge University Press, Cambridge (2002).

[17] A. Clark and L. Sadun, When size matters: Subshifts and their related tiling spaces, Ergodic Th. E6 Dynam. Syst. 23 (2003), 1043-57; arXiv:math.DS/0201152.

[18] J. Dieudonné, Foundations of Modern Analysis, vol. I, Academic Press, New York (1969).

[19] J.H. Elton, An ergodic theorem for iterated maps, Ergodic Th. ES Dynam. Syst. 7 (1987), 481-488.

[20] M. Fekete, Über die Verteilung der Wurzeln bei gewissen algebraischen Gleichungen mit ganzzahligen Koeffizienten, Math. Z. 17 (1923), 228-249.

[21] H. Furstenberg, Recurrence in Ergodic Theory and Combinatorial Number Theory, Princeton Univ. Press, Princeton, NJ (1981).

[22] C. Godrèche and J.M. Luck, Quasiperiodicity and randomness in tilings of the plane, J. Stat. Phys. 55 (1989), 1-28.

[23] P. Gohlke, On a Family of Semi-Compatible Random Substitutions, Masters Thesis, Bielefeld University (2017).

[24] A. Hof, On diffraction by aperiodic structures, Commun. Math. Phys. 169 (1995), 25-43.

[25] C. Huck and C. Richard, On pattern entropy of weak model sets, Discr. Comput. Geom. 54 (2015), 741-757; arXiv:1412.6307.

[26] B. Jessen and A. Wintner, Distribution functions and the Riemann zeta function, Trans. Amer. Math. Soc. 38 (1935), 48-88.

[27] G. Keller and C. Richard, Dynamics on the graph of the torus parametrisation, Ergodic Th. ES Dynam. Syst. 38 (2018) 1048-1085; arXiv:1511.06137.

[28] G. Keller, Maximal equicontinuous generic factors and weak model sets, preprint arXiv:1610.03998.

[29] J.-Y. Lee, R.V. Moody and B. Solomyak, Pure point dynamical and diffraction spectra, Ann. H. Poincaré 3 (2002), 1003-1018; arXiv:0910.4809.

[30] D. Lenz, Continuity of eigenfunctions of uniquely ergodic dynamical systems and intensity of Bragg peaks, Commun. Math. Phys. 287 (2009) 225-258; arXiv:math-ph/0608026.

[31] C. Lütkehölter, Diffraktion stochastischer Fibonacci-Mengen, Diploma thesis, Univ. Bielefeld (2010).

[32] M. Moll, On a Family of Random Noble Means Substitutions, PhD thesis, Univ. Bielefeld (2013); available at https://pub.uni-bielefeld.de/publication/2637807.

[33] M. Moll, Diffraction of random noble means words, J. Stat. Phys. 156 (2014), 1221-1236; arXiv: 1404.7411.

[34] R.V. Moody, Mathematical quasicrystals: A tale of two topologies, in XIVth International Congress on Mathematical Physics, ed. J.-C. Zambrini, World Scientific, Singapore (2006), pp. 68-77. 
[35] R.V. Moody and N. Strungaru, Point sets and dynamical systems in the autocorrelation topology, Bull. Can. Math. Soc. 47 (2004), 82-99.

[36] J. Nilsson, On the entropy of a family of random substitutions, Monatsh. Math. 166 (2012), 1-15; arXiv: 1103.4777.

[37] K.J. Palmer, Bifurcations, chaos and fractals, in Nonlinear Dynamics and Chaos, eds. R.L. Dewar and B.I. Henry, World Scientific, Singapore (1992), pp. 91-133.

[38] M. Queffélec, Substitution Dynamical Systems - Spectral Analysis, LNM 1294, 2nd ed., Springer, Berlin (2010).

[39] W. Rudin, Fourier Analysis on Groups, Wiley, New York (1962).

[40] D. Rust and T. Spindeler, Dynamical systems arising from random substitutions, Indag. Math., to appear; arXiv:1707.09836.

[41] T. Spindeler, Diffraction intensities of a class of binary Pisot substitutions via exponential sums, Monatsh. Math. 182 (2017), 143-153; arXiv:1608.01969

[42] T. Spindeler, Spectral Theory of Random Inflation Systems, PhD thesis, Univ. Bielefeld (2018); available at https://pub.uni-bielefeld.de/publication/2917383.

[43] N. Strungaru, Almost periodic pure point measures, in Aperiodic Order. Vol. 2: Crystallography and Almost Periodicity, eds. M. Baake and U. Grimm, Cambridge University Press, Cambridge (2017), pp. 271-342.

[44] K.R. Wicks, Fractals and Hyperspaces, LNM 1492, Springer, Berlin (1991).

FAKUltät FÜr Mathematik, Universität Bielefeld,

Postfach 100131, 33501 Bielefeld, Germany

E-mail address: \{mbaake,tspindel\}@math.uni-bielefeld.de

Department of Mathematical Sciences, MacEwan University,

10700104 Avenue, Edmonton, AB, Canada T5J 4S2

E-mail address: strungarun@macewan.ca 